\title{
Topological sensitivity analysis in fluorescence optical tomography
}

\author{
A. Laurain ${ }^{1}$, M. Hintermüller ${ }^{1,2}$, M. Freiberger ${ }^{3}$ and H. Scharfetter ${ }^{3}$ \\ ${ }^{1}$ Humboldt-University of Berlin, Department of Mathematics, Johann-von-Neumann-Haus, \\ Rudower Chaussee 25, D-12489 Berlin-Adlershof, Germany. \\ ${ }^{2}$ Karl-Franzens University of Graz, Department of Mathematics and Scientific Computing, \\ Heinrichstraße 36, $8010 \mathrm{Graz}$, Austria. \\ ${ }^{3}$ Institute of Medical Engineering, TU Graz, Kronesgasse 5 / II, 8010 Graz, Austria. \\ Emails: laurain@math.hu-berlin.de, hint@math.hu-berlin.de, \\ manuel.freiberger@tugraz. at and hermann. scharfetter@tugraz.at
}

\begin{abstract}
Fluorescence tomography is a non-invasive imaging modality that reconstructs fluorophore distributions inside a small animal from boundary measurements of fluorescence light. The associated inverse problem is stabilized by a priori properties or information. In this paper cases are considered where the fluorescent inclusions are well separated from the background and have a spatially constant concentration. Under these a priori assumptions, the identification process may be formulated as a shape optimization problem, where the interface between the fluorescent inclusion and the background constitutes the unknown shape. In this paper, we focus on the computation of the so-called topological derivative for fluorescence tomography which could be used as a stand-alone tool for the reconstruction of the fluorophore distributions or as the initialization in a level-set based method for determining the shape of the inclusions.
\end{abstract}

AMS subject classifications 49Q10, 49N45, 78M35.

Key words fluorescence optical tomography, inverse problem, topological derivative, asymptotic analysis

\section{Introduction}

Nowadays, fluorescence is widely used in medical sciences with applications ranging from gene sequencing to the distinction between healthy brain tissue and malign tumors. In pre-clinical sciences, it is often desired to track, e.g., tumor growth or the evolution of physiological parameters over time. Besides their non-destructive nature, optical techniques involving fluorescence are especially advantageous as there are numerous fluorophores available which bind specifically to certain tissue types or change their optical parameters in dependence on the surrounding biological milieu, e.g. the $\mathrm{pH}$-value $[20,35]$ or the tissue oxygenation $[33,40]$. 
For in-vivo applications, simple planar imaging methods are not suitable as the heavy scattering of the photons in biological tissue prevents the resolution of the depth and size of a fluorescent inclusion. This is sought to be mitigated by fluorescence tomography, where a series of light sources is placed on the surface of the sample and the emitted fluorescence light is measured from different projections. Compared to conventional modalities such as magnetic resonance imaging or $\mathrm{x}$-ray computed tomography, fluorescence tomography (or fluorescence diffuse optical tomography; FDOT for short) provides the advantages of the availability of numerous functionalized, i.e. milieu-dependent, fluorophores and the avoidance of ionizing radiation, a core requirement for longitudinal tumor assessment, for example.

Conventional image reconstruction in fluorescence tomography is based on the computation of the so-called sensitivity, which is the linearization of the forward problem with respect to small changes in fluorophore parameters. Recently, a number of authors addressed the inverse problem, i.e., the identification of the fluorophore concentration from boundary measurements of the photon flux, directly. Concerning the stabilization of the identification process several regularization techniques such as Tikhonov regularization within a Levenberg-Marquardt context, the Newton-Landweber method or Newton-CG algorithm as well as Gauß-Newton methods have been applied [2, 16, 19, 28, 29, 37, 38, 39].

In special applications, a-priori information on a localization of the fluorophore inside the biological specimen is available. This could be the case, for instance, when the fluorescent agent binds specifically to tumor tissue and the volume of the tumor has to be determined. Mathematically, this corresponds to invoking piecewise constant model parameters, i.e., fluorophore concentrations.

In the piecewise constant framework, we are looking for a partitioning $\left\{\Omega_{i}\right\}_{i=0}^{n_{c}}$ of the domain $\Omega$, with associated concentrations $\left\{c_{i}\right\}_{i=0}^{n_{c}}$ and $n_{c} \in \mathbb{N}$. The problem, thus, becomes a shape optimization problem, where the unknown shapes are the domains $\Omega_{i}$. For the numerical solution of this problem shape sensitivity calculus, as developed in [42], may be employed. Given $\left\{\Omega_{i}\right\}$, the values $c_{i}, i=0, . ., n_{c}$, are determined through standard minimization techniques in $\mathbb{R}^{n_{c}}$.

Such a shape optimization approach was successfully implemented for the electrical impedance tomography problem in [24, 25], for instance. In the latter work, the subregions were initialized by using the concept of topological sensitivity [41]. Then shape sensitivity [42] was used to locally improve the shape of the identified distributions, thus, providing a good estimate of the correct boundary of the object of interest.

In this paper we focus on the topological sensitivity part of the above mentioned procedure. In contrast to typical problems of shape optimization, different levels of topological differentiation must be computed, which leads to algorithmical challenges. The rest of the paper is organized as follows. In Section 2 we formulate the forward and inverse problems, respectively, and introduce the piecewise constant model. In Section 3 , the asymptotic expansions with respect to changes in the fluorophore concentrations of the perturbed solution is computed. This expansion is used in Section 4 to compute the topological derivative of the functional. Section 5 establishes the topological expansion in the domain dependent case, and in Section 6 the numerical algorithm is discussed and numerical results are provided. 


\section{Problem formulation}

\subsection{Forward model}

Nowadays, at least three models are commonly used for light propagation in biological tissues: the random-walk Monte-Carlo method [23], the radiative transport equation (RTE) [9] and the diffusion approximation [9]. The latter can be derived from the RTE assuming simplifying constraints such as scattering-dominant tissue, an isotropic scattering phase function and several constraints on the variation of optical parameters [9]. The model is of special interest due to its convenient structure. For fluorescence applications, two diffusion approximations, one for the excitation light and one for the light emitted by the fluorophore, may be coupled [30, 36].

In this paper, we consider what has been termed the full model in [16] where the optical parameters in the governing equation, i.e. the diffusion and absorption coefficients, are dependent on the fluorophore concentration. For the mathematical formulation of the forward problem, let $\Omega$ be a smooth ( $\mathcal{C}^{\infty}$-domain) bounded domain in $\mathbb{R}^{N}, N \geq 2$ and $\Sigma:=\partial \Omega$. By $n$ we denote the outward unit normal vector to $\Omega$. The forward problem under consideration consists in finding the solution $\left(\phi_{x}, \phi_{m}\right)$ to the coupled system

$$
\begin{aligned}
-\operatorname{div}\left(\kappa_{x} \nabla \phi_{x}\right)+\mu_{x} \phi_{x} & =q \quad \text { in } \Omega, \\
\kappa_{x} \partial_{n} \phi_{x}+\rho_{x} \phi_{x} & =0 \quad \text { on } \Sigma, \\
-\operatorname{div}\left(\kappa_{m} \nabla \phi_{m}\right)+\mu_{m} \phi_{m} & =\gamma \phi_{x} \quad \text { in } \Omega, \\
\kappa_{m} \partial_{n} \phi_{m}+\rho_{m} \phi_{m} & =0 \quad \text { on } \Sigma,
\end{aligned}
$$

where $\phi_{j}$ denotes the photon density at the excitation $(j=x)$ and emission wavelengths $(j=m)$. This system involves several (known) parameters. In fact, $\kappa_{j}, \mu_{j}$, $j=x, m$, depend on the fluorophore concentration $c$ through

$$
\kappa_{j}(c)=\left[N\left(\mu_{a, j}+\epsilon_{j} c+\mu_{s, j}^{\prime}\right)\right]^{-1}, \quad \mu_{j}(c)=\mu_{a, j}+\epsilon_{j} c+\frac{i \omega}{\nu} .
$$

Also, $\gamma$ depends on $c$ via the relation

$$
\gamma(c)=\frac{\eta}{1+i \omega \tau} \epsilon_{x} c .
$$

The absorption coefficient $\mu_{j}$ consists of the tissue's intrinsic absorption $\mu_{a, j}$ and the additional absorption $\epsilon_{j} c$ at locations where a fluorophore is present. The molar extinction coefficient $\epsilon_{j}$ relates the fluorophore concentration $c$ to its absorption and is assumed to be independent of the concentration. The reduced scattering coefficient is $\mu_{s, j}^{\prime}$. We denote by $\kappa_{j}$ the diffusion coefficient and $\rho_{j}$ the reflection coefficient of the light at the boundary of the domain. The fluorescent agent is quantified by its quantum yield $\eta$ and the fluorescent life-time $\tau$. We assume a collimated beam $q$ for the excitation light whose intensity is harmonically modulated with a frequency $\omega$. Finally, $\nu$ is the speed of light inside the sample. In section 6 numerical values for these coefficients are given.

As noted above, the forward problem consists in determining the complex amplitudes $\phi_{x}, \phi_{m}$ for a given distribution of the fluorophore and a prescribed set of excitations. The coefficients appearing in (5)-(6) are assumed to be known. To ensure existence of solutions to (1)-(4), we consider the following set of admissible fluorophore concentrations

$$
C_{a d}:=\left\{c \in L^{2}(\Omega): 0 \leq c \leq \bar{c}\right\} \subset L^{2}(\Omega)
$$


for some given $\bar{c}>0$; see [16] for details. For a given excitation $q$ in (1), the mathematical forward problem is formulated as the action of an operator

$$
F: C_{a d} \subset L^{2}(\Omega) \rightarrow L^{2}(\Sigma), c \mapsto \rho_{m} \phi_{m},
$$

which assigns to some prescribed fluorophore concentration $c$ the corresponding emitted light (photon flux) at the boundary.

\subsection{Inverse problem}

In experiments, the measurable quantity, i.e. phase and intensity of the emitted light at wavelength $\lambda_{m}$ on the boundary, is given by the photon flux at the boundary and is defined as

$$
\mathscr{M}:=-\kappa_{m} \partial_{n} \phi_{m}=\rho_{m} \phi_{m} \quad \text { on } \Sigma ;
$$

see [9] for details. The aim of fluorescence optical tomography is to determine an a priori unknown fluorophore concentration $c$ from measurements of the emitted light $\mathscr{M}$. For a single excitation $q$ in (1), this amounts to solving the equation

$$
F(c)=\mathscr{M}
$$

for $c \in C_{a d}$ with given $\mathscr{M} \in L^{2}(\Sigma)$.

A common approach to solve (8) is to minimize the squared $L^{2}$-distance on the boundary $\Sigma$ between $F(c)$ and $\mathscr{M}$ by means of an iterative procedure for computing $c$. Note that this parameter identification problem is ill-posed and, thus, requires appropriate regularization. Here, in our least-squares context we consider the objective

$$
J(c)=\|F(c)-\mathscr{M}\|_{L^{2}(\Sigma)}^{2}+\alpha R(c),
$$

where $R(c)$ denotes a function with a regularizing effect in the minimization process and $\alpha>0$ the associated regularization parameter. In [16, 19], for instance, $R(c)=$ $\|c\|_{L^{2}(\Omega)}^{2}$, the so-called Tikhonov regularization, is used.

In this paper we assume that the fluorescent inclusion has a sharp interface, and is a piecewise constant function, i.e., there are a background concentration $c_{0}>0$ and $n_{c}$ inclusions (i.e., possibly multiply connected sets) which have concentrations $c_{i} \neq c_{0}, i=1, \ldots, n_{c}$. Based on this assumption, $\Omega$ can be split into $n_{c}+1$ disjoint domains $\Omega_{0}$ and $\Omega_{i}$ with concentrations $c_{0}$ and $c_{i}, i=1, . ., n_{c}$, respectively. This yields $\Omega=\bigcup_{i=0}^{n_{c}} \Omega_{i}$ with $\Sigma \cap \Gamma_{i}=\emptyset$ for $i \geq 1$, where $\Gamma_{i}=\partial \Omega_{i}$. We then have $c=\sum_{i=0}^{n_{c}} c_{i} \mathbb{1}_{\Omega_{i}}$, where $\mathbb{1}_{\Omega_{i}}$ denotes the characteristic function of $\Omega_{i} \subset \Omega$.

In this framework, an appropriate regularization is given by the total variation seminorm

$$
R(c)=\int_{\Omega}|D c|=\sum_{i=1}^{n_{c}}\left|c_{0}-c_{i}\right| P\left(\Omega_{i}\right) .
$$

where $P\left(\Omega_{i}\right)$ stands for the perimeter of the set $\Omega_{i}$. Hence, in contrast to the Tikhonov regularization mentioned above, the total variation regularization imposes a certain regularity of the boundaries of the inclusions. With this regularization and introducing the notation

$$
\mathcal{J}_{s}\left(\left\{\Omega_{i}, c_{i}\right\}_{i=1}^{n_{c}}\right):=\int_{\Sigma}\left|F\left(c\left(\left\{\Omega_{i}, c_{i}\right\}_{i=1}^{n_{c}}\right)\right)-\mathscr{M}\right|^{2}+\alpha \sum_{i=1}^{n_{c}}\left|c_{0}-c_{i}\right| P\left(\Omega_{i}\right),
$$


the optical tomography problem is reduced to solving the following minimization problem which depends only on $\Omega_{i}$ and on the scalar values $c_{i}, i=1, . ., n_{c}$ :

$$
\begin{aligned}
& \operatorname{minimize} \mathcal{J}_{s}\left(\left\{\Omega_{i}, c_{i}\right\}_{i=1}^{n_{c}}\right) \\
& \quad \text { over } \Omega_{i} \subset \Omega \text { and } c_{i} \in \mathbb{R}, i=1, . ., n_{c} .
\end{aligned}
$$

Fixing $\Omega_{i}, i=1, . ., n_{c}$, for the moment and minimizing with respect to $c_{i}, i=1, . ., n_{c}$, we obtain $c_{i}=c_{i}\left(\left\{\Omega_{k}\right\}_{k=0}^{n_{c}}\right)$ and (10) can be reduced to

$$
\begin{aligned}
& \operatorname{minimize} \hat{\mathcal{J}}_{s}\left(\left\{\Omega_{i}\right\}_{i=1}^{n_{c}}\right):=\mathcal{J}_{s}\left(\left\{\Omega_{i}, c_{i}\left(\left\{\Omega_{k}\right\}_{k=0}^{n_{c}}\right)\right\}_{i=1}^{n_{c}}\right) \\
& \quad \text { over } \Omega_{i} \subset \Omega, i=1, \ldots, n_{c}
\end{aligned}
$$

Observe that (11) is a shape optimization problem, where the unknowns are the subsets $\Omega_{i}, i=1, . ., n_{c}$. As this problem is highly nonlinear, one needs to employ appropriate iterative solvers for its numerical solution. Particular instances are descent schemes relying on the shape gradient [24] of $\hat{\mathcal{J}}_{s}$ or the shape Hessian [26]. It is well-known that the performance of these solvers crucially depends on their initialization, which, in principle, is a difficult task as it needs to guess the topological distribution of the concentrations $c_{i}$ in $\Omega$.

Recently, the concept of topological derivative has attracted considerable attention in mathematical imaging and inverse problems; see [3, 4, 5, 6, 7, 8, 10, 11, 13, 15, 18, $22,24,25]$. Indeed it was found that topological sensitivities are ideal for computing reliable initial guesses without the need of inserting expert knowledge through userinteractive initialization. In this paper, we extend the topological sensitivity concept to fluorescence diffusive optical tomography in order to establish a reliable initialization mechanism for iteratively solving (11). Observe that, compared to electrical impedance tomography (EIT), the situation here is considerably more involved. First, FDOT requires the coupling of two elliptic partial differential equations and, secondly, the dependence of the states of the system on the wanted coefficient is significantly more nonlinear; compare (5)-(6) for the latter.

While the repeated application of the topological sensitivity concept yields an iterative algorithm for solving (11) in its own right, we here merely focus on the initialization, i.e. the first step, of a shape and topological sensitivity based solver for (11). It turns out that this procedure represents a reliable tool for detecting small regions $\Omega_{i}$ with concentrations different from the background concentration $c_{0}$. Subsequently, one may apply shape sensitivity in order to locally adjust for the size and shape of the sets $\Omega_{i}$.

In order to focus on the initialization step, we choose $\alpha=0$ and consider $c_{i}, i=$ $1, . ., n_{c}$, fixed. We note that the regularization may be computed in a subsequent shape optimization phase. Usually, we have more than one measurement at our disposal. Consequently, we study the problem

$$
\begin{aligned}
& \operatorname{minimize} \mathcal{J}\left(\left\{\Omega_{i}\right\}_{i=1}^{n_{c}}\right)=\sum_{k=1}^{n_{k}} \int_{\Sigma}\left|F\left(c\left(\left\{\Omega_{i}\right\}_{i=1}^{n_{c}}\right)\right)-\mathscr{M}_{k}\right|^{2} \\
& \quad \text { over } \Omega_{i} \subset \Omega, i=1, . ., n_{c}
\end{aligned}
$$

where $n_{k}$ is the number of measurements. In this paper, we take $n_{k}=1$ in (12) to simplify the presentation as the extension of our results to $n_{k}>1$ is rather straightforward; see $[24,25]$, for instance, in the context of EIT. 


\section{Topological derivative}

Singular perturbations of domains, where partial differential equations (PDEs) are posed, were first considered from a theoretical point of view in [27, 34] and later from an applied perspective through the so-called "Bubble method" [17], where a parameterized setting was used and holes were created in the domain according to a certain criterion. This idea was further developed by Sokolowski and Zochowski [41] as well as Guillaume and Masmoudi [21] by introducing the topological derivative.

The topological derivative measures the variation of an objective functional depending on a domain, when a small change in the topology of this domain occurs, for instance through the creation of a small hole or inclusion of any shape. For a mathematical description of this process, let $\Omega$ be an open set in $\mathbb{R}^{N}, B(\hat{y}, \delta)$ the open ball of radius $\delta>0$ centered at $\hat{y} \in \Omega$, and define $\Omega^{\delta}:=\Omega \backslash \overline{B(\hat{y}, \delta)}$. If the objective functional $J(\Omega)$ is differentiable with respect to the creation of the small hole $B(\hat{y}, \delta)$ as $\delta \rightarrow 0$, then one obtains the expansion

$$
J\left(\Omega^{\delta}\right)=J(\Omega)+\rho(\delta) T(\hat{y})+\mathcal{O}(\rho(\delta)), \quad \delta \rightarrow 0,
$$

with $\rho(\delta) \rightarrow 0$ and $\mathcal{O}(\rho(\delta)) / \rho(\delta) \rightarrow 0$ as $\delta \rightarrow 0$. The quantity $T(\hat{y})$ is called the topological derivative of $J$ at $\hat{y}$.

Here, we adapt this notion which was originally introduced for structural optimization problems to the FDOT problem. In FDOT we do not create "holes" as in structural optimization, but rather consider local perturbations $c_{\delta}$ of the type

$$
c_{\delta}=c_{0} \mathbb{1}_{\Omega^{\delta}}+\sum_{i=1}^{n_{c}} c_{i} \mathbb{1} \frac{}{B_{i}^{\delta}},
$$

with

$$
\Omega^{\delta}:=\Omega \backslash\left(\cup_{i=1}^{n_{c}} \overline{B_{i}^{\delta}}\right) \quad \text { and } \quad B_{i}^{\delta} \cap B_{j}^{\delta}=\varnothing \quad \text { for } \quad i \neq j,
$$

where $B_{i}^{\delta}$ is an open ball of radius $\delta$ and center $\hat{y}_{i} \in \Omega$ which is associated with the given concentration $c_{i}, i=1, . ., n_{c}$. Therefore, we are in the framework of section 2.2, with $\Omega^{\delta}$ and $B_{i}^{\delta}$ corresponding to $\Omega_{0}$ and $\Omega_{i}$, respectively, and we seek an expansion similar to (13) in order to locate the regions of given concentration $c_{i}, i=1, . ., n_{c}$.

We shall assume that the points $\hat{y}_{i} \in \Omega$ which determine the location of the inclusions satisfy

$$
\begin{aligned}
& 0<\hat{d} \leq\left|\hat{y}_{i}-\hat{y}_{j}\right|, \forall i \neq j, \\
& 0<\hat{d} \leq \operatorname{dist}\left(\hat{y}_{i}, \Sigma\right), i=1, . ., n_{c}
\end{aligned}
$$

for some given positive real number $\hat{d}$. The radius $\delta$ should also be small enough so that the distance of $B_{i}^{\delta}$ to the boundary $\Sigma$ and the other inclusions is always larger than $\hat{d} / 2$. The assumption (16)-(17) is used to obtain a pointwise estimate for the remainder $\mathcal{O}(\rho(\delta))$ in (13). A similar assumption was made for the inverse conductivity problem in [14]. In Section 4.2 we discuss the behaviour of the remainder $\mathcal{O}(\rho(\delta))$ when the inclusion is close to or even on the boundary. We remark that (16)-(17) is related to the fact that the remainder $\mathcal{O}(\rho(\delta))$ in (13) has different scales depending on whether $\hat{y}_{i}$ is asymptotically close to the boundary or at a fixed distance from it. The former behaviour perturbs the detection process in the whole domain by a bias towards the boundary. In order to compensate for this effect, we consider higher-order terms in 
the expansion of $J\left(\Omega^{\delta}\right)$ which are concentrated on the boundary; see Section 4.2 for details.

In general we could assume that the domain $\Omega$ is perturbed by introducing several simultaneous inclusions, i.e. $n_{c}>1$. However, as it was shown for the EIT-problem in [24], it is enough to consider only the case $n_{c}=1$ for the topological expansions. The case of several inclusions can be straightforwardly obtained by summing up the contributions of single inclusions considered separately in the topological derivative.

In view of (14), the coefficients in (5)-(6) also have the decompositions

$$
\begin{aligned}
\kappa_{j}\left(c_{\delta}\right) & =\kappa_{j}\left(c_{0}\right) \mathbb{1}_{\Omega^{\delta}}+\kappa_{j}\left(c_{1}\right) \mathbb{1}_{\overline{B^{\delta}}}, \\
\mu_{j}\left(c_{\delta}\right) & =\mu_{j}\left(c_{0}\right) \mathbb{1}_{\Omega^{\delta}}+\mu_{j}\left(c_{1}\right) \mathbb{1}_{\overline{B^{\delta}}}, \\
\gamma\left(c_{\delta}\right) & =\gamma\left(c_{0}\right) \mathbb{1}_{\Omega^{\delta}}+\gamma\left(c_{1}\right) \mathbb{1}_{\overline{B^{\delta}}},
\end{aligned}
$$

respectively. The notation $\kappa_{j, 0}, \kappa_{j, 1}$ will be used later instead of $\kappa_{j}\left(c_{0}\right), \kappa_{j}\left(c_{1}\right)$ and a similar notation is employed for $\mu_{j}$ and $\gamma$.

We now proceed with the asymptotic expansion of $\mathcal{J}\left(\left\{B_{i}^{\delta}\right\}_{i=1}^{n_{c}}\right)$ in (12) with respect to $\delta$. Eventually, this provides the topological derivative of $\mathcal{J}$ in the case of fixed $\left(c_{0}, c_{1}\right)$. In the present framework, we decompose $\left(\phi_{x}^{\delta}, \phi_{m}^{\delta}\right)$, defined as the solutions of (1)-(4) with the concentration $c_{\delta}$, as follows

$$
\phi_{x}^{\delta}=\phi_{x, 0}^{\delta} \mathbb{1}_{\Omega^{\delta}}+\phi_{x, 1}^{\delta} \mathbb{1}_{\overline{B^{\delta}}}, \quad \phi_{m}^{\delta}=\phi_{m, 0}^{\delta} \mathbb{1}_{\Omega^{\delta}}+\phi_{m, 1}^{\delta} \mathbb{1}_{\overline{B^{\delta}}} .
$$

Actually, we may significantly simplify the situation (and the notation, as well) by noting that $\phi_{x}$ and $\phi_{m}$ satisfy structurally the same equation but with different parameter values and right-hand sides. In the case of $\phi_{m}$, the right-hand side depends on $c$ through $\gamma$ and $\phi_{x}$. Once we have an expansion of $\phi_{x}^{\delta}$, we may plug this expansion (with adapted data) into the equation for $\phi_{m}^{\delta}$. Therefore, in what follows, we consider first the following general problem

$$
\begin{aligned}
-\operatorname{div}(\kappa(c) \nabla \phi)+\mu(c) \phi & =f & & \text { in } \Omega, \\
\kappa(c) \partial_{n} \phi+\rho \phi & =0 & & \text { on } \Sigma .
\end{aligned}
$$

We assume in this section that $f$ depends on $\delta$ in the following way

$$
f=f^{\delta}=f_{0} \mathbb{1}_{\Omega^{\delta}}+f_{1} \mathbb{1}_{\overline{B^{\delta}}},
$$

where

$$
f_{0} \in \mathcal{C}^{2}(\Omega) \text { and } f_{1} \in \mathcal{C}^{4}(\Omega)
$$

are independent of $\delta$. As in (21), the solution $\phi^{\delta}$ of (22)-(23) with $c_{\delta}$ instead of $c$, is split into

$$
\phi^{\delta}=\phi_{0}^{\delta} \mathbb{1}_{\Omega^{\delta}}+\phi_{1}^{\delta} \mathbb{1}_{\overline{B^{\delta}}},
$$

where $\left(\phi_{0}^{\delta}, \phi_{1}^{\delta}\right)$ satisfy the coupled system

$$
\begin{aligned}
-\kappa_{0} \Delta \phi_{0}^{\delta}+\mu_{0} \phi_{0}^{\delta} & =f_{0} \quad \text { in } \Omega^{\delta}, \\
\kappa_{0} \partial_{n} \phi_{0}^{\delta}+\rho \phi_{0}^{\delta} & =0 \quad \text { on } \Sigma, \\
-\kappa_{1} \Delta \phi_{1}^{\delta}+\mu_{1} \phi_{1}^{\delta} & =f_{1} \quad \text { in } B^{\delta}, \\
\kappa_{0} \partial_{n} \phi_{0}^{\delta} & =\kappa_{1} \partial_{n} \phi_{1}^{\delta} \quad \text { on } \Gamma^{\delta}, \\
\phi_{0}^{\delta} & =\phi_{1}^{\delta} \quad \text { on } \Gamma^{\delta},
\end{aligned}
$$

with $n$ the outward unit normal vector to $\partial \Omega^{\delta}$. Further we have $\Gamma^{\delta}:=\partial B^{\delta}, \kappa_{0}:=$ $\kappa\left(c_{0}\right), \kappa_{1}:=\kappa\left(c_{1}\right), \mu_{0}:=\mu\left(c_{0}\right)$, and $\mu_{1}:=\mu\left(c_{1}\right)$. 


\subsection{Preliminary description of the asymptotic expansion}

In a first step towards computing the topological sensitivity of $\mathcal{J}$ in (12), we aim at an asymptotic expansion of $\phi^{\delta}=\phi_{0}^{\delta} \mathbb{1}_{\Omega^{\delta}}+\phi_{1}^{\delta} \mathbb{1}_{\overline{B^{\delta}}}$ of the type

$$
\begin{aligned}
& \phi_{0}^{\delta}(y)=\phi^{0}(y)+U_{0}(z)+V_{0}(z)+w_{0}^{\delta}(y) \quad \text { in } \Omega^{\delta}, \\
& \phi_{1}^{\delta}(y)=\sum_{i=0}^{2} \phi_{1}^{(i)}(y)+\widetilde{\phi}_{1}+U_{1}(z)+V_{1}(z)+w_{1}^{\delta}(y) \quad \text { in } B^{\delta} .
\end{aligned}
$$

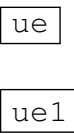

Here $y$ stands for the so-called slow variable in the domain $\Omega$, and $z:=\delta^{-1}(y-\hat{y})$ for the fast variable in the unbounded domain $\mathbb{R}^{N} \backslash \overline{B^{1}}$, with $B^{1}$ the ball of radius 1 and center 0 , the origin of a Cartesian coordinate system. The notions fast and slow variables refer to the rapid growth of $z$ compared to $y$ away from $\hat{y}$ due to the factor $\delta^{-1}$, tending to infinity as $\delta \rightarrow 0$. Here, $\phi^{0}$ is a term of regular type, whereas

$$
U=U_{0} \mathbb{1}_{\mathbb{R}^{N} \backslash \overline{B^{1}}}+U_{1} \mathbb{1}_{\overline{B^{1}}} \text { and } V=V_{0} \mathbb{1}_{\mathbb{R}^{N} \backslash \overline{B^{1}}}+V_{1} \mathbb{1}_{\overline{B^{1}}}
$$

are terms of boundary layer type, expressed in the variable $z$ and defined in $\mathbb{R}^{N}$. Terms of boundary layer type are usually concentrated in a neighbourhood of the inclusion $B^{\delta}$, in the sense that they vanish quickly when moving away from the inclusion.

The structure of the asymptotic expansion (30) comes from general results on asymptotic expansions originally considered in [27, 34]. In this paper, we use the method of compound asymptotic expansions as introduced in [34]. The first term $\phi^{0}$ of the expansion (30) is the solution of (25)-(29) for $\delta=0$ and corresponds to the unperturbed problem. As indicated above, the difference $\phi_{0}^{\delta}-\phi^{0}$ is concentrated in a neighbourhood of $B^{\delta}$ and approximated at the first and second order by the boundary layers $U$ and $V$, respectively.

The boundary layers $U$ and $V$ leave in turn discrepancies on the outer boundary $\Sigma$ and in $\Omega$, which are compensated by the remainder $w^{\delta}:=w_{0}^{\delta} \mathbb{1}_{\Omega^{\delta}}+w_{1}^{\delta} \mathbb{1}_{\overline{B^{\delta}}}$, which may be further expanded similarly to $\phi^{\delta}$, as it is explained in [34]. This asymptotic procedure also requires $U_{0}$ and $V_{0}$ to be harmonic functions.

In the case of the expansion (31), the approximating functions $\phi_{1}^{(i)}, i=0,1,2$, are given explicitly, since we work on the simple domain $B^{\delta}$. Finally pointwise estimates can be obtained for $w^{\delta}$ following [34].

\subsection{First term of regular type}

In what follows, the notation $D^{k} \phi(\hat{y}) \cdot(y-\hat{y})^{k}$ represents the derivative of order $k$ of a given function $\phi$ at $\hat{y}$ in direction $y-\hat{y}$. The first approximation of $\phi^{\delta}$ in $\Omega^{\delta}$ is $\phi^{0}$, the so-called first term of regular type which satisfies

$$
\begin{aligned}
-\kappa_{0} \Delta \phi^{0}+\mu_{0} \phi^{0} & =f_{0} \quad \text { in } \Omega, \\
\kappa_{0} \partial_{n} \phi^{0}+\rho \phi^{0} & =0 \quad \text { on } \Sigma .
\end{aligned}
$$

The regularity $f_{0} \in \mathcal{C}^{2}(\Omega)$ and the smoothness of $\Omega$ imply $\phi^{0} \in \mathcal{C}^{4}(\Omega)$. Further we introduce the remainder

$$
\psi_{0}^{\delta}:=\phi_{0}^{\delta}-\phi^{0} \quad \text { in } \Omega^{\delta}
$$


Using $f_{1}$ and $\phi^{0} \in \mathcal{C}^{4}(\Omega)$ we have the following Taylor expansions at $\hat{y} \in \Omega$ :

$$
\begin{aligned}
& \phi^{0}(y)=\sum_{k=0}^{2} D^{k} \phi^{0}(\hat{y}) \cdot(y-\hat{y})^{k}+\widetilde{\phi}^{0}(y), \\
& f_{1}(y)=\sum_{k=0}^{2} D^{k} f_{1}(\hat{y}) \cdot(y-\hat{y})^{k}+\widetilde{f}_{1}(y),
\end{aligned}
$$

for $|y-\hat{y}|$ sufficiently small, where $\widetilde{\phi}^{0}, \widetilde{f}_{1}$ denote the remainders at $\hat{y}$ depending on $\phi^{0}, f_{1}$. Plugging (36) into (27) and (35) into $\phi_{1}^{\delta}=\phi_{0}^{\delta}=\phi^{0}+\psi_{0}^{\delta}$ on $\Gamma^{\delta}$ yields

$$
\begin{aligned}
-\kappa_{1} \Delta \phi_{1}^{\delta}(y)+\mu_{1} \phi_{1}^{\delta}(y) & =\sum_{k=0}^{2} D^{k} f_{1}(\hat{y}) \cdot(y-\hat{y})^{k}+\widetilde{f}_{1}(y) \quad \text { in } B^{\delta}, \\
\phi_{1}^{\delta}(y) & =\sum_{k=0}^{2} D^{k} \phi^{0}(\hat{y}) \cdot(y-\hat{y})^{k}+\widetilde{\phi}^{0}(y)+\psi_{0}^{\delta}(y) \text { on } \Gamma^{\delta} .
\end{aligned}
$$

Therefore, we make the following ansatz for $\phi_{1}^{\delta}$ :

$$
\phi_{1}^{\delta}=\sum_{i=0}^{2} \phi_{1}^{(i)}+\widetilde{\phi}_{1}+\psi_{1}^{\delta} \quad \text { in } B^{\delta},
$$

where $\phi_{1}^{(k)}, k=0,1,2$ and $\widetilde{\phi}_{1}$ satisfy

$$
\begin{aligned}
-\kappa_{1} \Delta \phi_{1}^{(k)}+\mu_{1} \phi_{1}^{(k)} & =D^{k} f_{1}(\hat{y}) \cdot(y-\hat{y})^{k} & & \text { in } B^{\delta}, \\
\phi_{1}^{(k)} & =D^{k} \phi^{0}(\hat{y}) \cdot(y-\hat{y})^{k} & & \text { on } \Gamma^{\delta},
\end{aligned}
$$

and

$$
\begin{aligned}
-\kappa_{1} \Delta \widetilde{\phi}_{1}+\mu_{1} \widetilde{\phi}_{1} & =\widetilde{f}_{1}(y) \quad \text { in } B^{\delta}, \\
\widetilde{\phi}_{1} & =\widetilde{\phi}^{0}(y) \quad \text { on } \Gamma^{\delta} .
\end{aligned}
$$

The remainder $\psi_{1}^{\delta}$ in (39) has similar properties; see (47)-(48) below. Note that $\phi_{1}^{(k)}, k=$ $0,1,2$, and $\widetilde{\phi}_{1}$ depend on $\delta$, although for simplicity this is not apparent in the notation.

In view of (28) and (34) we have

$$
-\kappa_{0} \partial_{n} \psi_{0}^{\delta}=-\kappa_{1} \partial_{n} \phi_{1}^{\delta}+\kappa_{0} \partial_{n} \phi^{0} \quad \text { on } \Gamma^{\delta} .
$$

The solution $\phi_{1}^{\delta}$ actually depends on $\psi_{0}^{\delta}$ through $\psi_{1}^{\delta}$ in (38). In (44), using (39) and (35), one may thus replace $\phi_{1}^{\delta}$ to obtain (49) below. Finally, gathering (37), (38) and (39), the remainders $\left(\psi_{0}^{\delta}, \psi_{1}^{\delta}\right)$ satisfy the following system

$$
\begin{aligned}
-\kappa_{0} \Delta \psi_{0}^{\delta}+\mu_{0} \psi_{0}^{\delta} & =0 & & \text { in } \Omega^{\delta}, \\
\kappa_{0} \partial_{n} \psi_{0}^{\delta}+\rho \psi_{0}^{\delta} & =0 & & \text { on } \Sigma,
\end{aligned}
$$




\subsubsection{Computation of $\phi_{1}^{(k)}$}

The functions $\phi_{1}^{(k)}, k=0,1,2$, in (39) may be computed explicitely by using polar or spherical coordinates for $N=2$ or 3 , respectively, and separation of variables. They are expressed in terms of Bessel functions of the first kind in two dimensions (commonly denoted by $J_{k}$ ) and of the first spherical Bessel function (also known as sinc function commonly denoted by $j_{k}$ ) in three dimensions. Here we write $s_{k}$ for these Bessel functions regardless of the dimension; see [43] for details on Bessel functions. We directly provide the results here and defer detailed calculations to the appendix. In fact, we have

$$
\begin{aligned}
& \phi_{1}^{(0)}=c_{1}^{(0)} s_{0}(\lambda r)+\frac{f_{1}(\hat{y})}{\mu_{1}}, \\
& \text { with } c_{1}^{(0)}=\left[\phi^{0}(\hat{y})-\frac{f_{1}(\hat{y})}{\mu_{1}}\right] s_{0}(\lambda \delta)^{-1} \text { and } \lambda=\left(-\frac{\mu_{1}}{\kappa_{1}}\right)^{1 / 2} \\
& \phi_{1}^{(1)}=s_{1}(\lambda r) c_{1}^{(1)} \cdot n+\frac{\nabla f_{1}(\hat{y}) \cdot(y-\hat{y})}{\mu_{1}}, \\
& \quad \text { with } c_{1}^{(1)}=\delta\left[\frac{\nabla f_{1}(\hat{y})}{\mu_{1}}-\nabla \phi^{0}(\hat{y})\right] s_{1}(\lambda \delta)^{-1} . \\
& \phi_{1}^{(2)}=c_{1}^{(2)} s_{0}(\lambda r) \\
& \quad+\frac{\delta^{2}}{4} s_{4}(\lambda r) s_{4}(\lambda \delta)^{-1}\left[-\frac{1}{N} \Delta\left(\phi^{0}-\frac{f_{1}}{\mu_{1}}\right)(\hat{y})+D^{2}\left(\phi^{0}-\frac{f_{1}}{\mu_{1}}\right)(\hat{y}) \cdot(n)^{2}\right] \\
& \quad+\frac{1}{2 \mu_{1}} D^{2} f_{1}(\hat{y}) \cdot(y-\hat{y})^{2}+\frac{\kappa_{1}}{\left(\mu_{1}\right)^{2}} \Delta f_{1}(\hat{y}) \\
& \quad \text { with } c_{1}^{(2)}=\left[\frac{\delta^{2}}{4} \Delta \phi^{0}(\hat{y})-\frac{\delta^{2}}{4 \mu_{1}} \Delta f_{1}(\hat{y})-\frac{\kappa_{1}}{\left(\mu_{1}\right)^{2}} \Delta f_{1}(\hat{y})\right] s_{0}(\lambda \delta)^{-1} .
\end{aligned}
$$

In view of (49), we need the normal derivatives of $\phi_{1}^{(k)}, k=0,1,2$, at $r=\delta$ given by the asymptotic formulae as $\delta \rightarrow 0$ :

$$
\begin{aligned}
\partial_{n} \phi_{1}^{(0)}(\delta) & =-c_{1}^{(0)} \lambda s_{0}^{\prime}(\lambda \delta)=\left[\phi^{0}(\hat{y})-\frac{f_{1}(\hat{y})}{\mu_{1}}\right]\left(\lambda^{2} \frac{\delta}{N}\right)+d_{1}^{(0)}, \\
\partial_{n} \phi_{1}^{(1)}(\delta) & =\nabla \phi^{0}(\hat{y}) \cdot n+d_{1}^{(1)}, \\
\partial_{n} \phi_{1}^{(2)}(\delta) & =\delta N^{-1} \Delta \phi^{0}(\hat{y})-\delta D^{2} \phi^{0}(\hat{y}) \cdot(n)^{2}+d_{1}^{(2)}, \\
\partial_{n} \widetilde{\phi}_{1}(\delta) & =\widetilde{d}_{1} .
\end{aligned}
$$

with $d_{1}^{(0)}=\mathcal{O}\left(\delta^{3}\right), d_{1}^{(1)}=\mathcal{O}\left(\delta^{2}\right), d_{1}^{(2)}=\mathcal{O}\left(\delta^{3}\right)$ and $\widetilde{d}_{1}=\mathcal{O}\left(\delta^{2}\right)$.

\subsubsection{Expansion of the remainder}

Inserting (53)-(56) into (49) we obtain

$$
\begin{aligned}
& -\kappa_{0} \partial_{n} \psi_{0}^{\delta}+\kappa_{1} \partial_{n} \psi_{1}^{\delta} \\
& =\delta^{0}\left[\left(\kappa_{0}-\kappa_{1}\right) \nabla \phi^{0}(\hat{y}) \cdot n\right] \\
& +\delta^{1}\left[-\frac{\kappa_{1} \lambda^{2}}{N}\left(\phi^{0}(\hat{y})-\frac{f_{1}(\hat{y})}{\mu_{1}}\right)+\kappa_{1}\left(-\frac{1}{N} \Delta \phi^{0}(\hat{y})+D^{2} \phi^{0}(\hat{y}) \cdot(n)^{2}\right)\right. \\
& \left.\quad-\kappa_{0} D^{2} \phi^{0}(\hat{y}) \cdot(n)^{2}\right]+d_{1}^{n} \quad \text { on } \Gamma^{\delta},
\end{aligned}
$$


where

$$
d_{1}^{n}:=\kappa_{0} \partial_{n} \widetilde{\phi}^{0}-\kappa_{1}\left(d_{1}^{(0)}+d_{1}^{(1)}+d_{1}^{(2)}+\widetilde{d}_{1}\right)=\mathcal{O}\left(\delta^{2}\right) .
$$

Note that for the sake of reference we indicate the zero-order term in $\delta$ by $\delta^{0}$ and the first-order term by $\delta^{1}$. According to (32) we have

$$
\frac{\kappa_{0}}{N} \Delta \phi^{0}(\hat{y})-\frac{\mu_{0}}{N} \phi^{0}(\hat{y})+\frac{f_{0}(\hat{y})}{N}=0 .
$$

Thus, we may add (59) to the $\delta^{1}$-term in (57). Using $\lambda^{2}=-\mu_{1} / \kappa_{1}$ this leads us to

$$
\begin{aligned}
-\kappa_{0} \partial_{n} \psi_{0}^{\delta}+\kappa_{1} \partial_{n} \psi_{1}^{\delta}= & \delta^{0}\left[\left(\kappa_{0}-\kappa_{1}\right) \nabla \phi^{0}(\hat{y}) \cdot n\right] \\
& +\delta^{1}\left[\frac{f_{0}(\hat{y})-f_{1}(\hat{y})}{N}+\frac{\mu_{1}-\mu_{0}}{N} \phi^{0}(\hat{y})\right. \\
& \left.\quad+\left(\kappa_{0}-\kappa_{1}\right)\left(\frac{\Delta \phi^{0}(\hat{y})}{N}-D^{2} \phi^{0}(\hat{y}) \cdot(n)^{2}\right)\right] \\
& +d_{1}^{n} \quad \text { on } \Gamma^{\delta} .
\end{aligned}
$$

Summarizing, (45)-(48) and (60) establish an asymptotic expansion for the equation satisfied by the remainder $\psi^{\delta}=\psi_{0}^{\delta} \mathbb{1}_{\Omega^{\delta}}+\psi_{1}^{\delta} \mathbb{1}_{\overline{B^{\delta}}}$, given by (45)-(48) and (60).

The next step in the expansion consists in approximating the remainder using boundary layers.

\subsection{Boundary layers}

\subsubsection{First boundary layer}

Following the discussion in [34, Section 2.2] and the procedure described in Section 3.1 , we introduce the first boundary layer $U$ which compensates the term of order $\delta^{0}$ in (60):

$$
U(z)=U_{0}(z) \mathbb{1}_{\mathbb{R}^{N} \backslash \overline{B^{1}}}(z)+U_{1}(z) \mathbb{1}_{\overline{B^{1}}}(z), \quad z \in \mathbb{R}^{N},
$$

where $z=\delta^{-1}(y-\hat{y})$ is the fast variable as described in section 3.1. The functions $U_{0}$ and $U_{1}$ satisfy

$$
\begin{aligned}
-\Delta U_{0} & =0 \quad \text { in } \mathbb{R}^{N} \backslash \overline{B^{1}} \\
-\kappa_{0} \partial_{n} U_{0}+\kappa_{1} \partial_{n} U_{1} & =\left(\kappa_{0}-\kappa_{1}\right) \nabla \phi^{0}(\hat{y}) \cdot n \quad \text { on } \Gamma^{1}:=\partial B^{1} \\
-\Delta U_{1} & =0 \quad \text { in } B^{1} \\
U_{1} & =U_{0} \quad \text { on } \Gamma^{1} \\
U_{0}(z) & \rightarrow 0 \quad \text { as }|z| \rightarrow \infty .
\end{aligned}
$$

The solution pair of (61)-(65) is given by

$$
\begin{aligned}
& U_{0}\left(\delta^{-1}(y-\hat{y})\right)=\frac{\delta^{N}}{|y-\hat{y}|^{N}} \beta_{1} \nabla \phi^{0}(\hat{y}) \cdot(y-\hat{y}), \\
& U_{1}\left(\delta^{-1}(y-\hat{y})\right)=\beta_{1} \nabla \phi^{0}(\hat{y}) \cdot(y-\hat{y}),
\end{aligned}
$$

with

$$
\beta_{1}=\frac{\kappa_{0}-\kappa_{1}}{\kappa_{1}+(N-1) \kappa_{0}} .
$$


We check that $U$ is indeed a solution of (61)-(65). First of all, $U_{0}, U_{1}$ are clearly harmonic. Therefore (61) and (63) are satisfied. Further,

$$
\begin{aligned}
& \left.\partial_{n} U_{0}\right|_{\Gamma^{1}}=-\left.\partial_{r} U_{0}\right|_{\Gamma^{1}}=(1-N) \beta_{1} \nabla \phi^{0}(\hat{y}) \cdot n \\
& \left.\partial_{n} U_{1}\right|_{\Gamma^{1}}=\beta_{1} \nabla \phi^{0}(\hat{y}) \cdot n .
\end{aligned}
$$

This, finally, leads to

$$
-\kappa_{0} \partial_{n} U_{0}+\kappa_{1} \partial_{n} U_{1}=\left(-\kappa_{0}(1-N) \beta_{1}+\kappa_{1} \beta_{1}\right) \nabla \phi^{0}(\hat{y}) \cdot n \quad \text { on } \Gamma^{1},
$$

which proves that (66)-(67) are solutions of (61)-(64) with (65) obviously satisfied.

\subsubsection{Second boundary layer}

Similarly we introduce a second boundary layer $V$ to compensate the term $\delta^{1}$ in (60):

$$
V(z)=V_{0}(z) \mathbb{1}_{\mathbb{R}^{N} \backslash \overline{B^{1}}}(z)+V_{1}(z) \mathbb{1}_{\overline{B^{1}}}(z), \quad z \in \mathbb{R}^{N} .
$$

We decompose $V_{0}, V_{1}$ into $V_{0}=V_{0}^{(1)}+V_{0}^{(2)}$ and $V_{1}=V_{1}^{(1)}+V_{1}^{(2)}$ which solve:

$$
\begin{aligned}
&-\Delta V_{0}^{(1)}=0 \quad \text { in } \mathbb{R}^{N} \backslash \overline{B^{1}}, \\
&-\kappa_{0} \partial_{n} V_{0}^{(1)}+\kappa_{1} \partial_{n} V_{1}^{(1)}=\delta\left[\frac{f_{0}(\hat{y})-f_{1}(\hat{y})}{N}+\frac{\mu_{1}-\mu_{0}}{N} \phi^{0}(\hat{y})\right] \quad \text { on } \Gamma^{1}, \\
&-\Delta V_{1}^{(1)}=0 \quad \text { in } B^{1}, \quad V_{1}^{(1)}=V_{0}^{(1)} \quad \text { on } \Gamma^{1} .
\end{aligned}
$$

and

$$
-\Delta V_{0}^{(2)}=0 \quad \text { in } \mathbb{R}^{N} \backslash \overline{B^{1}},
$$

$$
-\kappa_{0} \partial_{n} V_{0}^{(2)}+\kappa_{1} \partial_{n} V_{1}^{(2)}=\delta\left(\kappa_{0}-\kappa_{1}\right)\left(\frac{\Delta \phi^{0}(\hat{y})}{N}-D^{2} \phi^{0}(\hat{y}) \cdot(n)^{2}\right) \text { on } \Gamma^{1} \text {, }
$$

$$
-\Delta V_{1}^{(2)}=0 \quad \text { in } B^{1}, \quad V_{1}^{(2)}=V_{0}^{(2)} \quad \text { on } \Gamma^{1} .
$$$$
V_{0}^{(2)}(z) \rightarrow 0 \text { as }|z| \rightarrow 0 \text {. }
$$

In dimension $N=3$, we need the condition

$$
V_{0}^{(1)}(z) \rightarrow 0 \quad \text { as }|z| \rightarrow \infty,
$$

which yields uniqueness of the solution to (71)-(73). In dimension $N=2$, (78) cannot be satisfied since the solution is logarithmic. Therefore, instead we impose

$$
V_{0}^{(1)}(z)=0 \text { on } \Gamma_{1} \text { for } N=2 .
$$

Consequently, we obtain the solutions

$$
\begin{aligned}
& V_{0}^{(1)}:=\beta_{2} \delta^{N} Q(|y-\hat{y}|), \\
& V_{0}^{(2)}:=\beta_{3} \kappa_{0} \delta^{N+2}\left[\frac{\Delta \phi^{0}(\hat{y})}{N|y-\hat{y}|^{N}}-\frac{D^{2} \phi^{0}(\hat{y}) \cdot(y-\hat{y})^{2}}{|y-\hat{y}|^{N+2}}\right],
\end{aligned}
$$


where $\beta_{2}, \beta_{3}$ are constants to be determined and $Q$ is a singular function which depends on the dimension:

$$
\begin{aligned}
& Q(|y-\hat{y}|)=-\ln \left(\delta^{-1}|y-\hat{y}|\right) \quad \text { for } N=2, \\
& Q(|y-\hat{y}|)=|y-\hat{y}|^{-1} \quad \text { for } N=3 .
\end{aligned}
$$

For $V_{0}^{(1)}$ we readily deduce that $V_{1}^{(1)} \equiv 0$ and $\partial_{n} V_{1}^{(1)} \equiv 0$ on $\Gamma^{1}$. Since $\partial_{n}=-\partial_{r}$ with $r=|y-\hat{y}|$ on $\Gamma^{1}$, we find

$$
\beta_{2}=\frac{1}{\kappa_{0} N}\left[f_{1}(\hat{y})-f_{0}(\hat{y})+\left(\mu_{0}-\mu_{1}\right) \phi^{0}(\hat{y})\right] .
$$

In view of (76) and (80) we obtain

$$
V_{1}^{(2)}=\beta_{3} \kappa_{0}\left[\frac{\Delta \phi^{0}(\hat{y})}{N} r^{2}-D^{2} \phi^{0}(\hat{y}) \cdot(y-\hat{y})^{2}\right] .
$$

Equation (75) leads to

$$
\beta_{3}=\frac{\kappa_{1}-\kappa_{0}}{\kappa_{0}\left(2 \kappa_{1}+N \kappa_{0}\right)} .
$$

\subsection{Asymptotic expansion and remainder}

In view of the approximation of $\phi^{\delta}$ computed in sections 3.2 and 3.3 we introduce the remainders

$$
\begin{aligned}
& w_{0}^{\delta}:=\phi_{0}^{\delta}-\left(\phi^{0}+U_{0}+V_{0}\right) \quad \text { in } \Omega^{\delta}, \\
& w_{1}^{\delta}:=\phi_{1}^{\delta}-\left(\sum_{i=0}^{2} \phi_{1}^{(i)}+\widetilde{\phi}_{1}+U_{1}+V_{1}\right) \quad \text { in } B^{\delta} .
\end{aligned}
$$

Thus, we have obtained the expansions in (30)-(31). Due to (60) we infer

$$
\kappa_{0} \partial_{n} w_{0}^{\delta}-\kappa_{1} \partial_{n} w_{1}^{\delta}=\kappa_{0} \partial_{n}\left(\psi_{0}^{\delta}-U_{0}-V_{0}\right)-\kappa_{1} \partial_{n}\left(\psi_{1}^{\delta}-U_{1}-V_{1}\right)=-d_{1}^{n}
$$

Therefore the remainders $w_{0}^{\delta}$ and $w_{1}^{\delta}$ satisfy

$$
\begin{aligned}
-\kappa_{0} \Delta w_{0}^{\delta}+\mu_{0} w_{0}^{\delta} & =-\mu_{0}\left(U_{0}+V_{0}\right) \quad \text { in } \Omega^{\delta}, \\
\kappa_{0} \partial_{n} w_{0}^{\delta}+\rho w_{0}^{\delta} & =-\kappa_{0} \partial_{n}\left(U_{0}+V_{0}\right)+\rho_{0}\left(U_{0}+V_{0}\right) \quad \text { on } \Sigma, \\
-\kappa_{1} \Delta w_{1}^{\delta}+\mu_{1} w_{1}^{\delta} & =\widetilde{f}_{1}-\mu_{1}\left(U_{1}+V_{1}\right) \text { in } B^{\delta}, \\
w_{0}^{\delta}-w_{1}^{\delta} & =\widetilde{\phi}^{0} \quad \text { on } \Gamma^{\delta}, \\
\kappa_{0} \partial_{n} w_{0}^{\delta}-\kappa_{1} \partial_{n} w_{1}^{\delta} & =-d_{1}^{n} \quad \text { on } \Gamma^{\delta},
\end{aligned}
$$

Adapting the results from [34, chapter 2.2], using assumptions (16)-(17) and in view of the estimate (58) and of the definition of $U, V, \widetilde{f}_{1}, \widetilde{\phi}_{0}$ one can prove the pointwise estimate

$$
w^{\delta}(y):=w_{0}^{\delta} \mathbb{1}_{\Omega^{\delta}}+w_{1}^{\delta} \mathbb{1}_{\overline{B^{\delta}}}=\mathcal{O}\left(\delta^{N}\right) \quad \text { for } y \in \bar{\Omega} \text { and } \hat{y} \in \Omega .
$$




\section{Computation of the topological derivative}

\subsection{Domain-independent right-hand side}

In this section we work under the assumptions (16)-(17). For one inclusion $\left(n_{c}=1\right)$ and fixed scalar values $c_{0}$ and $c_{1},(12)$ may be written as

$$
\mathcal{J}\left(\Omega^{\delta}\right)=\int_{\Sigma}\left|F\left(c_{\delta}\right)-\mathscr{M}\right|^{2}=\int_{\Sigma}\left|\rho \phi_{0}^{\delta}-\mathscr{M}\right|^{2},
$$

where $\mathscr{M}$ denotes a single boundary measurement. Plugging (34) into (94) we obtain

$$
\mathcal{J}\left(\Omega^{\delta}\right)=\mathcal{J}(\Omega)+K^{\delta}+L^{\delta},
$$

with the notation

$$
\begin{aligned}
K^{\delta} & :=\mathcal{R} e\left(\int_{\Sigma} 2 \rho \psi_{0}^{\delta} \overline{\left(\rho \phi^{0}-\mathscr{M}\right)}\right) \\
L^{\delta} & :=\int_{\Sigma}\left|\rho \psi_{0}^{\delta}\right|^{2} .
\end{aligned}
$$

where $\bar{z}$ and $\mathcal{R} e(z)$ denote the complex conjugate and the real part of a complex number $z$, respectively.

For computing the topological derivative of $\mathcal{J}$ we introduce the adjoint state $p$ satisfying

$$
\begin{aligned}
-\kappa_{0} \Delta p+\mu_{0} p & =0 \quad \text { in } \Omega, \\
\kappa_{0} \partial_{n} p+\rho p & =2 \overline{\left(\rho \phi^{0}-\mathscr{M}\right)} \quad \text { on } \Sigma .
\end{aligned}
$$

We now recast $K^{\delta}$ as an integral over $\Gamma^{\delta}$. In fact, using (99) we get

$$
K^{\delta}=\mathcal{R} e\left(\int_{\Sigma} \rho \psi_{0}^{\delta}\left(\kappa_{0} \partial_{n} p+\rho p\right)\right)=\mathcal{R} e\left(\rho \kappa_{0} \int_{\Sigma} \psi_{0}^{\delta} \partial_{n} p+\rho^{2} \int_{\Sigma} \psi_{0}^{\delta} p\right) .
$$

Using Green's formula in $\Omega^{\delta}$, we compute the first integral in (100):

$$
\int_{\Sigma} \psi_{0}^{\delta} \partial_{n} p=\int_{\Omega_{\delta}} \psi_{0}^{\delta} \Delta p-p \Delta \psi_{0}^{\delta}+\int_{\Sigma} p \partial_{n} \psi_{0}^{\delta}-\int_{\Gamma^{\delta}} \psi_{0}^{\delta} \partial_{n} p-p \partial_{n} \psi_{0}^{\delta}
$$

Combining (45), (46), (98) and (101) yields

$$
\kappa_{0} \int_{\Sigma} \psi_{0}^{\delta} \partial_{n} p=-\int_{\Sigma} \rho \psi_{0}^{\delta} p-\kappa_{0} \int_{\Gamma^{\delta}} \psi_{0}^{\delta} \partial_{n} p-p \partial_{n} \psi_{0}^{\delta},
$$

such that

$$
K^{\delta}=\mathcal{R} e\left(-\rho \kappa_{0} \int_{\Gamma^{\delta}} \psi_{0}^{\delta} \partial_{n} p-p \partial_{n} \psi_{0}^{\delta}\right) .
$$

In view of (30), (66)-(67), (79)-(80) and the local regularity of $p$, using a Taylor expansion of $p$ at $\hat{y}$ and the estimate (93), we obtain for the first boundary integral in (103)

$$
\int_{\Gamma^{\delta}} \psi_{0}^{\delta} \partial_{n} p=\int_{\Gamma^{\delta}}\left(U_{0}+V_{0}+w_{0}^{\delta}\right) \partial_{n} p=-\left|B^{\delta}\right| \beta_{1} \nabla \phi^{0}(\hat{y}) \cdot \nabla p(\hat{y})+R_{K}^{(1)}
$$


where

$$
R_{K}^{(1)}=\mathcal{O}\left(\delta^{2 N-1}\right)
$$

For the other integral in (103) we have

$$
\int_{\Gamma_{\delta}} p \partial_{n} \psi_{0}^{\delta}=\int_{\Gamma_{\delta}} p \partial_{n}\left(U_{0}+V_{0}^{(1)}+V_{0}^{(2)}+w_{0}^{\delta}\right) .
$$

With $y-\hat{y}=-\delta n$ on $\Gamma_{\delta}$ and using a Taylor expansion of $p$ about $\hat{y}$ we get

$$
\begin{aligned}
\int_{\Gamma_{\delta}} p \partial_{n} U_{0} & =-\delta(1-N) \int_{\Gamma_{\delta}} \nabla p(\hat{y}) \cdot n\left[\beta_{1} \nabla \phi^{0}(\hat{y}) \cdot n\right]+R_{K}^{(2)} \\
& =\delta(N-1) \frac{\left|B^{\delta}\right|}{\delta} \beta_{1} \nabla \phi^{0}(\hat{y}) \cdot \nabla p(\hat{y})+R_{K}^{(2)} \\
\int_{\Gamma^{\delta}} p \partial_{n} V_{0}^{(1)} & =-\int_{\Gamma^{\delta}} p \partial_{r} V_{0}^{(1)}=\frac{\beta_{2} \delta^{N}}{\delta^{N-1}} \int_{\Gamma^{\delta}} p \\
& =\beta_{2} \delta^{N} p(\hat{y}) \frac{\left|\Gamma^{\delta}\right|}{\delta^{N-1}}+R_{K}^{(3)}, \\
\int_{\Gamma^{\delta}} p \partial_{n} V_{0}^{(2)} & =R_{K}^{(4)}
\end{aligned}
$$

where $R_{K}^{(2)}=\mathcal{O}\left(\delta^{N+2}\right), R_{K}^{(3)}=\mathcal{O}\left(\delta^{N+2}\right)$ and $R_{K}^{(4)}=\mathcal{O}\left(\delta^{N+2}\right)$. Thus, in view of (83), (107), and since $\delta\left|\Gamma^{\delta}\right|=N\left|B^{\delta}\right|$ we find

$$
\begin{aligned}
K^{\delta}= & \mathcal{R} e\left(\rho \kappa_{0} N\left|B^{\delta}\right| \beta_{1} \nabla \phi^{0}(\hat{y}) \cdot \nabla p(\hat{y})\right) \\
& +\mathcal{R} e\left(\rho\left|B^{\delta}\right|\left[\left(\mu_{0}-\mu_{1}\right) \phi^{0}(\hat{y})+\left(f_{1}(\hat{y})-f_{0}(\hat{y})\right)\right] p(\hat{y})\right)+R_{K},
\end{aligned}
$$

where $R_{K}:=\sum_{i=1}^{4} R_{K}^{(i)}=\mathcal{O}\left(\delta^{2 N-1}\right)$.

The above computations prove the following result.

thm1 Theorem 1 Assume $\hat{y}$ satisfies the assumptions (16)-(17). Then the functional in (94) admits the expansion

$$
\mathcal{J}\left(\Omega^{\delta}\right)=\mathcal{J}(\Omega)+\left|B^{\delta}\right| T(\hat{y})+R^{\delta}(\hat{y}) \text { as } \delta \searrow 0,
$$

where $T$ is the so-called topological derivative given by

$$
\begin{aligned}
& T(\hat{y})=\mathcal{R} e\left(\rho \kappa_{0} N \beta_{1} \nabla \phi^{0}(\hat{y}) \cdot \nabla p(\hat{y})\right. \\
&\left.+\rho\left[\left(\mu_{0}-\mu_{1}\right) \phi^{0}(\hat{y})+f_{1}(\hat{y})-f_{0}(\hat{y})\right] p(\hat{y})\right),
\end{aligned}
$$

with

$$
\beta_{1}=\frac{\kappa_{0}-\kappa_{1}}{\kappa_{1}+(N-1) \kappa_{0}},
$$

and $R^{\delta}(\hat{y}):=R_{K}(\hat{y})+L^{\delta}(\hat{y})=\mathcal{O}\left(\delta^{2 N-1}\right)$.

\subsection{Higher-order terms}

When the hidden inclusion has a moderate size, an asymptotically small trial inclusion close to the boundary may produce boundary values which better match the measurements than an asymptotically small trial inclusion even at the correct position inside the domain due to the ill-posed nature of the problem. This behavior is related to 
the non-uniformity of remainder $R^{\delta}$. The latter is also an issue in numerical practice where one typically introduces trial inclusions of small, but positive radius (rather then asymptotically vanishing quantities).

In order to remedy this effect and motivated by numerical practice, we consider trial inclusions of a certain size and compute higher-order terms in (109) for obtaining suitable topological expansions in this situation. One may even compute the full second-order expansion as it was done in [12, 25, 34]. This, however, requires a tedious analysis. Here, based on our experience in [24], we rather consider several "intermediate" higher-order terms only (but not the full second-order expansion), as the incorporation of these terms into the topological expansion already provides a sufficient correction of the boundary effect in the topological derivative.

In mathematical terms, this means that $L^{\delta}$ in (95) and consequently $R^{\delta}$ in (110) may have different $\delta$-scales depending on $\hat{y}$. Since $\psi_{0}^{\delta}=\phi_{0}^{\delta}-\phi^{0}=U_{0}+V_{0}+w_{0}^{\delta}$ in $\Omega^{\delta}$ and in view of (66), (79) and (80) we have $\psi_{0}^{\delta}(y)=\mathcal{O}\left(\delta^{N}\right)$ if $y \in \Sigma$ and $\hat{y} \notin \Sigma$ which leads to $L^{\delta}(\hat{y})=\mathcal{O}\left(\delta^{2 N}\right)$ for $\hat{y} \notin \Sigma$. However, $L^{\delta}(\hat{y})$ may have a lower $\delta$-order if $\hat{y} \in \Sigma$. To illustrate this effect, we describe a particular case for $N=2$. Assume that $\hat{y}=(0,0)$ and $\Omega$ is a square with one side being the segment

$$
\Sigma_{1}:=\left\{y=\left(y_{1}, y_{2}\right):\left|y_{1}\right| \leq 1, y_{2}=0\right\}
$$

Then we decompose

$$
\int_{\Sigma}|U|^{2}=\int_{\Sigma_{1}}|U|^{2}+\int_{\Sigma \backslash \Sigma_{1}}|U|^{2} .
$$

A short computation gives (with $N=2$ )

$$
\begin{aligned}
\int_{\Sigma_{1}}|U|^{2} & =\left|\partial_{y_{1}} \phi^{0}(\hat{y})\right|^{2} \beta_{1}^{2}\left(\delta^{2 N} \int_{\delta<\left|y_{1}\right| \leq 1} y_{1}^{2(1-N)} d y_{1}+\int_{\left|y_{1}\right| \leq \delta} y_{1}^{2} d y_{1}\right) \\
& =2 \beta_{1}^{2}\left|\partial_{y_{1}} \phi^{0}(\hat{y})\right|^{2}\left(\delta^{4}\left(\delta^{-1}-1\right)+\delta^{3} / 3\right)=\mathcal{O}\left(\delta^{3}\right) .
\end{aligned}
$$

Since $\hat{y} \notin \Sigma \backslash \Sigma_{1}$ we get $\int_{\Sigma \backslash \Sigma_{1}}|U|^{2}=\mathcal{O}\left(\delta^{4}\right)$. Therefore we have

$$
\int_{\Sigma}|U|^{2}=\mathcal{O}\left(\delta^{3}\right)
$$

A similar calculation shows that $\int_{\Sigma}|V|^{2}=\mathcal{O}\left(\delta^{3}\right)$. Thus we get $L^{\delta}(\hat{y})=\mathcal{O}\left(\delta^{2 N-1}\right)$ for $\hat{y} \in \Sigma$ and $N=2$ whereas $L^{\delta}(\hat{y})=\mathcal{O}\left(\delta^{2 N}\right)$ for $\hat{y} \notin \Sigma$. Performing a similar calculation for $N=3$ when $\Omega$ is a cube and $\hat{y} \in \Sigma$ we get $L^{\delta}(\hat{y})=\mathcal{O}\left(\delta^{2 N-2}\right)$. The case of a general smooth geometry $\Sigma$ gives a similar result.

For the remainder $R_{K}$ in (109) we have only shown the estimate $\mathcal{O}\left(\delta^{2 N-1}\right)$ due to (105), but one may actually obtain $R_{K}^{(1)}=\mathcal{O}\left(\delta^{2 N}\right)$ for $N=2$ by performing a higherorder expansion of $\mathcal{J}\left(\Omega^{\delta}\right)$. Therefore, $R_{K}$ is actually $\mathcal{O}\left(\delta^{N+2}\right)$. The rigorous proof of this statement would require a rather involved analysis which is beyond the scope of this paper. Our observation is justified by the behavior of the usual second-order topological expansions; see in particular [12, 25, 34].

Therefore in (95), $K^{\delta}$ contributes to $\mathcal{O}\left(\delta^{N}\right)$-terms (the topological derivative) and $\mathcal{O}\left(\delta^{N+2}\right)$ terms (the remainder $R_{K}$ ) whereas $L^{\delta}$ contributes to $\mathcal{O}\left(\delta^{N+1}\right)$ when $\hat{y} \in \Sigma$. Thus, in our numerical application we take into account $\mathcal{O}\left(\delta^{N+1}\right)$-terms of $L^{\delta}$ as a correction near the boundary for inclusions of moderate size rather than higher-order terms of the order $\mathcal{O}\left(\delta^{N+2}\right)$ coming from $K^{\delta}$. 
We extract the most significant terms from $L^{\delta}$ and use them in the topological expansion. In fact, $L^{\delta}$ in (97) may be decomposed into

$$
L^{\delta}(\hat{y})=L_{0}^{\delta}(\hat{y})+L_{1}^{\delta}(\hat{y})
$$

with

$$
L_{0}^{\delta}(\hat{y}):=\int_{\Sigma}\left|\rho\left(U+V^{(1)}\right)\right|^{2}, \quad L_{1}^{\delta}(\hat{y}):=L^{\delta}(\hat{y})-L_{0}^{\delta}(\hat{y}) .
$$

Instead of (110), we use the following expansion of $\mathcal{J}\left(\Omega^{\delta}\right)$ in our numerics

$$
\mathcal{J}\left(\Omega^{\delta}\right)=\mathcal{J}(\Omega)+\left|B^{\delta}\right| \mathcal{T}(\hat{y})+L_{0}^{\delta}(\hat{y})+\widetilde{R}^{\delta} \text { as } \delta \searrow 0
$$

with $\widetilde{R}^{\delta}:=R^{\delta}-L_{0}^{\delta}$.

\subsection{Domain-dependent right-hand side}

In Section 4 we have obtained the topological derivative of $\mathcal{J}$ depending on a function $\phi^{\delta}$ which solves (25)-(29). Choosing $f_{0}=f_{1}=q$, we obtain the asymptotic expansion of $\phi_{x}^{\delta}$. In the case of $\phi_{m}^{\delta}$, we choose $f_{0}=f_{0}^{\delta}:=\gamma_{0} \phi_{x}^{\delta}$ and $f_{1}=f_{1}^{\delta}:=\gamma_{1} \phi_{x}^{\delta}$. But we cannot in principle apply the result of Theorem 1 where we have assumed that $f_{0}$ and $f_{1}$ are independent of $\delta$. However, Theorem 1 will be used to obtain a part of the expansion of $\mathcal{J}$ depending on $\phi_{m}^{\delta}$.

In this chapter we consider (22)-(23) and replace $\phi$ by $\phi_{x}$ or $\phi_{m}$ and the corresponding right-hand sides. In fact, since we eventually take $f^{\delta}=\gamma\left(c_{\delta}\right) \phi_{x}^{\delta}$, we replace $f^{\delta}$ by a similar ansatz as in (30)-(31), i.e.,

$$
\begin{aligned}
& f_{0}^{\delta}=\gamma_{0} \phi_{x}^{0}+\gamma_{0}\left(U_{x, 0}+V_{x, 0}+w_{x, 0}^{\delta}\right) \quad \text { in } \Omega^{\delta} \\
& f_{1}^{\delta}=\gamma_{1}\left(\sum_{i=0}^{2} \phi_{x, 1}^{(i)}+\widetilde{\phi}_{x, 1}\right)+\gamma_{1}\left(U_{x, 1}+V_{x, 1}+w_{x, 1}^{\delta}\right) \quad \text { in } B^{\delta} .
\end{aligned}
$$

We decompose $f_{0}^{\delta}$ and $f_{1}^{\delta}$ into $\delta$-independent and $\delta$-dependent parts

$$
f_{0}^{\delta}=: f_{0}+\widetilde{f}_{0}^{\delta}, \quad f_{1}^{\delta}=: f_{1}+\widetilde{f}_{1}^{\delta}
$$

with

$$
f_{0}=\gamma_{0} \phi_{x}^{0}, \quad f_{1}=\gamma_{1} \sum_{i=0}^{1} \phi_{x, 1}^{(i, 0)} .
$$

The functions $\phi_{x, 1}^{(i, 0)}$ are the main part (independent on $\delta$ ) of $\phi_{x, 1}^{(i)}$, respectively. According to (50)-(52) and the expansions of $s_{0}$ and $s_{1}$ in the appendix, they are given by

$$
\begin{aligned}
\phi_{x, 1}^{(0,0)} & :=\left[\phi^{0}(\hat{y})-\frac{f_{1}(\hat{y})}{\mu_{1}}\right] s_{0}(\lambda r)+\frac{f_{1}(\hat{y})}{\mu_{1}}, \\
\phi_{x, 1}^{(1,0)} & :=s_{1}(\lambda r) \frac{N}{\lambda}\left[\frac{\nabla f_{1}(\hat{y})}{\mu_{1}}-\nabla \phi^{0}(\hat{y})\right] \cdot n+\frac{\nabla f_{1}(\hat{y}) \cdot(y-\hat{y})}{\mu_{1}} .
\end{aligned}
$$

In this way we can apply the results of Section 4 at least for the $\delta$-independent terms $f_{0}$ and $f_{1}$. Plugging (115) into the right-hand sides of (25) and (27) leads to considering the following ansatz for $\phi_{m}^{\delta}$ :

$$
\psi_{m, U}^{\delta}:=\phi_{m}^{\delta}-\phi_{m, \phi}^{\delta}
$$


where $\phi_{m, \phi}^{\delta}$ is defined as in (25)-(29), i.e.

$$
\begin{aligned}
-\operatorname{div}\left(\kappa_{m}\left(c_{\delta}\right) \nabla \phi_{m, \phi}^{\delta}\right)+\mu_{m}\left(c_{\delta}\right) \phi_{m, \phi}^{\delta} & =f_{0} \mathbb{1}_{\Omega^{\delta}}+f_{1} \mathbb{1}_{\overline{B^{\delta}}} \\
\kappa_{m}\left(c_{\delta}\right) \partial_{n} \phi_{m, \phi}^{\delta}+\rho_{m} \phi_{m, \phi}^{\delta} & =0 \quad \text { in } \Omega,
\end{aligned}
$$

The right-hand side in (120) satisfies the regularity assumptions (24). Note that

$$
\phi_{x, 1}^{(i)}-\phi_{x, 1}^{(i, 0)}=\mathcal{O}\left(\delta^{2}\right) \text { and } \sum_{i=0}^{1} \phi_{x, 1}^{(i, 0)}(\hat{y})=\phi^{0}(\hat{y}),
$$

and $\psi_{m, U}^{\delta}$ is the solution of

$$
\begin{aligned}
& -\operatorname{div}\left(\kappa_{m}\left(c_{\delta}\right) \nabla \psi_{m, U}^{\delta}\right)+\mu_{m}\left(c_{\delta}\right) \psi_{m, U}^{\delta} \\
& =\gamma_{0}\left(U_{x, 0}+V_{x, 0}+w_{x, 0}^{\delta}\right) \mathbb{1}_{\Omega^{\delta}}+\gamma_{1}\left(U_{x, 1}+V_{x, 1}+w_{x, 1}^{\delta}\right) \mathbb{1}_{\overline{B^{\delta}}} \\
& \quad+\gamma_{1}\left(\sum_{i=0}^{1}\left(\phi_{x, 1}^{(i)}-\phi_{x, 1}^{(0, i)}\right)+\phi_{x, 1}^{(2)}+\widetilde{\phi}_{x, 1}\right) \mathbb{1}_{\overline{B^{\delta}}} \quad \text { in } \Omega, \\
& \kappa_{m}\left(c_{\delta}\right) \partial_{n} \psi_{m, U}^{\delta}+\rho_{m} \psi_{m, U}^{\delta}=0 \quad \text { on } \Sigma .
\end{aligned}
$$

\subsubsection{Computation of the topological derivative}

The shape functional evaluated at the perturbed domain $\Omega^{\delta}$ is given by

$$
\mathcal{J}\left(\Omega^{\delta}\right)=\int_{\Sigma}\left|F\left(c_{\delta}\right)-\mathscr{M}\right|^{2}=\int_{\Sigma}\left|\rho_{m} \phi_{m}^{\delta}-\mathscr{M}\right|^{2} .
$$

Introducing $\psi_{m, \phi}^{\delta}:=\phi_{m, \phi}^{\delta}-\phi_{m}^{0}$ we obtain with (119)

$$
\phi_{m}^{\delta}=\phi_{m}^{0}+\psi_{m, \phi}^{\delta}+\psi_{m, U}^{\delta}
$$

which yields

$$
\mathcal{J}\left(\Omega^{\delta}\right)=\mathcal{J}(\Omega)+\mathcal{K}_{m, \phi}^{\delta}+\mathcal{K}_{m, U}^{\delta}+\mathcal{L}_{m}^{\delta}
$$

with the notations

$$
\begin{aligned}
\mathcal{K}_{m, \phi}^{\delta} & :=\mathcal{R} e\left(\int_{\Sigma} 2 \rho_{m} \psi_{m, \phi}^{\delta} \overline{\left(\rho_{m} \phi_{m}^{0}-\mathscr{M}\right)}\right), \\
\mathcal{K}_{m, U}^{\delta} & :=\mathcal{R} e\left(\int_{\Sigma} 2 \rho_{m} \psi_{m, U}^{\delta} \overline{\left(\rho_{m} \phi_{m}^{0}-\mathscr{M}\right)}\right), \\
\mathcal{L}_{m}^{\delta} & :=\int_{\Sigma}\left|\rho_{m}\left(\psi_{m, \phi}^{\delta}+\psi_{m, U}^{\delta}\right)\right|^{2} .
\end{aligned}
$$

To compute $\mathcal{K}_{m, \phi}^{\delta}$, one may apply Theorem 1 of Section 4.1. Replacing $f_{0}$ and $f_{1}$ in (111) by their expressions (116) leads to the formula

$$
\begin{aligned}
\mathcal{K}_{m, \phi}^{\delta}=\left|B^{\delta}\right| \mathcal{R} e\left(\rho_{m} N \kappa_{m, 0} \beta_{m, 1} \nabla \phi_{m}^{0}(\hat{y}) \cdot \nabla p_{m}(\hat{y})\right. & \left.\quad+\rho_{m}\left[\left(\mu_{m, 0}-\mu_{m, 1}\right) \phi_{m}^{0}(\hat{y})+\left(\gamma_{1}-\gamma_{0}\right) \phi_{x}^{0}(\hat{y})\right] p_{m}(\hat{y})\right) \\
& +\mathcal{R}_{\mathcal{K}_{m, \phi}}(\hat{y}),
\end{aligned}
$$

where $\mathcal{R}_{\mathcal{K}_{m, \phi}}$ is the remainder given by (109) and $\beta_{m, 1}$ is defined as in (68). 
On one hand, in view of (122) and (123) we have $\psi_{m, U}^{\delta}=\mathcal{O}\left(\delta^{N}\right)$ for all $\hat{y} \in \bar{\Omega}$. On the other hand $\psi_{m, \phi}^{\delta}(y)$ is also of the order $\mathcal{O}\left(\delta^{N}\right)$ when $\hat{y} \in \Omega$ but may have a lower order when $\hat{y} \in \Sigma$. Therefore, following the discussion in Section 4.2, we consider the decomposition

$$
\mathcal{L}_{m}^{\delta}=: \mathcal{L}_{0, m}^{\delta}+\mathcal{L}_{1, m}^{\delta}
$$

where

$$
\mathcal{L}_{0, m}^{\delta}(\hat{y}):=\int_{\Sigma}\left|\rho_{m}\left(U_{m}+V_{m}^{(1)}\right)\right|^{2}, \quad \mathcal{L}_{1, m}^{\delta}:=\mathcal{L}_{m}^{\delta}-\mathcal{L}_{0, m}^{\delta}=\mathcal{O}\left(\delta^{2 N}\right) .
$$

The boundary layers $U_{m}$ and $V_{m}^{(1)}$ come from the expansion of $\phi_{m, \phi}^{\delta}$ as in section 3 . The term $\mathcal{L}_{0, m}^{\delta}$ is then used for the numerical realization; see (157).

We employ the following decomposition

$$
\psi_{m, U}^{\delta}=: \psi_{m, U}^{(0)}+\psi_{m, U}^{(1)}
$$

where $\psi_{m, U}^{(0)}$ satisfies

$$
\begin{aligned}
-\kappa_{m, 0} \Delta \psi_{m, U}^{(0)}+\mu_{m, 0} \psi_{m, U}^{(0)}=\xi & \text { in } \Omega \\
\kappa_{m, 0} \partial_{n} \psi_{m, U}^{(0)}+\rho_{m} \psi_{m, U}^{(0)}=0 & \text { on } \Sigma
\end{aligned}
$$

with $\xi:=\xi_{0} \mathbb{1}_{\Omega^{\delta}}+\xi_{1} \mathbb{1} \frac{}{B^{\delta}}$ and

$$
\xi_{0}=\widetilde{f}_{0}^{\delta}, \quad \xi_{1}=\gamma_{1}\left(\phi_{x, 1}^{\delta}-\sum_{i=0}^{2} \phi_{x, 1}^{(i)}-\widetilde{\phi}_{x, 1}\right)
$$

Using (130) in (127), we get $\mathcal{K}_{m, U}^{\delta}=\mathcal{K}_{m, U}^{(0)}+\mathcal{K}_{m, U}^{(1)}$ with

$$
\mathcal{K}_{m, U}^{(i)}:=\mathcal{R} e\left(\int_{\Sigma} 2 \rho_{m} \psi_{m, U}^{(i)} \overline{\left(\rho_{m} \phi_{m}^{0}-\mathscr{M}\right)}\right), \quad i=0,1
$$

In other words we are considering the following expansion instead of (125):

$$
\mathcal{J}\left(\Omega^{\delta}\right)=\mathcal{J}(\Omega)+\mathcal{K}_{m, \phi}^{\delta}+\mathcal{K}_{m, U}^{(0)}+\widetilde{\mathcal{L}}_{m}^{\delta}
$$

where

$$
\widetilde{\mathcal{L}}_{m}^{\delta}=\mathcal{L}_{m}^{\delta}+\mathcal{K}_{m, U}^{(1)}
$$

The function $\psi_{m, U}^{(1)}$ satisfies a similar equation as (25)-(29). Therefore the leading term in $\mathcal{K}_{m, U}^{(i)}$ may be computed as in (109), with $\psi_{m, U}^{(0)}$ replacing $\phi^{0}$. In view of the definition of $\xi$ we have $\psi_{m, U}^{(0)}=\mathcal{O}\left(\delta^{2}\right)$ and thus we obtain $\mathcal{K}_{m, U}^{(1)}(y)=\mathcal{O}\left(\delta^{N+2}\right)$.

The coupled system of adjoint states $\left(p_{m}, p_{x}\right)$ is given by

$$
\begin{aligned}
-\kappa_{m, 0} \Delta p_{m}+\mu_{m, 0} p_{m} & =0 \quad \text { in } \Omega, \\
\kappa_{m, 0} \partial_{n} p_{m}+\rho_{m} p_{m} & =2 \overline{\left(\rho_{m} \phi_{m}^{0}-\mathscr{M}\right)} \quad \text { on } \Sigma, \\
-\kappa_{x, 0} \Delta p_{x}+\mu_{x, 0} p_{x} & =\gamma_{0} p_{m} \quad \text { in } \Omega \\
\kappa_{x, 0} \partial_{n} p_{x}+\rho_{x} p_{x} & =0 \quad \text { on } \Sigma .
\end{aligned}
$$


We start with the computation of an integral proportional to $\mathcal{K}_{m, U}^{(0)}$. Using the boundary condition for $p_{m}$ and Green's formula in $\Omega$ we obtain

$$
\begin{aligned}
\int_{\Sigma} 2 \psi_{m, U}^{(0)} \overline{\left(\rho_{m} \phi^{0}-\mathscr{M}\right)}=\int_{\Sigma} \psi_{m, U}^{(0)}\left(\kappa_{m, 0} \partial_{n} p_{m}+\rho_{m} p_{m}\right) \\
=\int_{\Omega} \kappa_{m, 0} \psi_{m, U}^{(0)} \Delta p_{m}-\kappa_{m, 0} p_{m} \Delta \psi_{m, U}^{(0)} \\
\quad+\int_{\Sigma}\left(\kappa_{m, 0} \partial_{n} \psi_{m, U}^{(0)}+\rho_{m} \psi_{m, U}^{(0)}\right) p_{m} \\
=\int_{\Omega} \mu_{0, m} p_{m} \psi_{m, U}^{(0)}-\mu_{0, m} p_{m} \psi_{m, U}^{(0)}+\xi p_{m}=\int_{\Omega} \xi p_{m}
\end{aligned}
$$

Using (135) and applying Green's formula in $\Omega^{\delta}$ yields

$$
\begin{aligned}
\gamma_{0} \int_{\Omega} \xi p_{m}= & \int_{\Omega^{\delta}} \xi_{0}\left(-\kappa_{x, 0} \Delta p_{x}+\mu_{x, 0} p_{x}\right)+\gamma_{0} \int_{B^{\delta}} \xi_{1} p_{m} \\
= & \int_{\Omega^{\delta}}-\kappa_{x, 0} p_{x} \Delta \xi_{0}+\mu_{x, 0} \xi_{0} p_{x}+\gamma_{0} \int_{B^{\delta}} \xi_{1} p_{m} \\
& +\int_{\Sigma} \kappa_{x, 0}\left(\partial_{n} \xi_{0} p_{x}-\xi_{0} \partial_{n} p_{x}\right)+\int_{\Gamma_{\delta}} \kappa_{x, 0}\left(\partial_{n} \xi_{0} p_{x}-\xi_{0} \partial_{n} p_{x}\right) .
\end{aligned}
$$

For the first integral in (142) we get

$\int_{\Omega^{\delta}}-\kappa_{x, 0} \Delta \xi_{0} p_{x}+\mu_{x, 0} \xi_{0} p_{x}=\gamma_{0} \int_{\Omega^{\delta}}\left[-\kappa_{x, 0} \Delta\left(\phi_{x}^{\delta}-\phi_{x}^{0}\right)+\mu_{x, 0}\left(\phi_{x}^{\delta}-\phi_{x}^{0}\right)\right] p_{x}=0$.

For the second integral we have

$$
\gamma_{0} \int_{B^{\delta}} \xi_{1} p_{m}=\gamma_{1} \gamma_{0} \int_{B^{\delta}}\left(U_{x, 1}+V_{x, 1}+w^{\delta}\right) p_{m} .
$$

Using a Taylor expansion of $p_{m}$ and the estimate (93) we get

$$
\int_{B^{\delta}}\left(U_{x, 1}+V_{x, 1}\right) p_{m}=\mathcal{O}\left(\delta^{N+2}\right) \quad \text { and } \quad \int_{B^{\delta}} w^{\delta} p_{m}=\mathcal{O}\left(\delta^{2 N}\right)
$$

which implies

$$
\gamma_{0} \int_{B^{\delta}} \xi_{1} p_{m}=\mathcal{O}\left(\delta^{N+2}\right) .
$$

For the first integral in (143) we get, using (2) and (136),

$$
\int_{\Sigma} \kappa_{x, 0}\left(\partial_{n} \xi_{0} p_{x}-\xi_{0} \partial_{n} p_{x}\right)=\int_{\Sigma}\left(\kappa_{x, 0} \partial_{n} \xi_{0}+\rho_{x} \xi_{0}\right) p_{x}=0
$$

For the second integral in (143), as in (104) we obtain

$$
-\int_{\Gamma_{\delta}} \kappa_{x, 0} \xi_{0} \partial_{n} p_{x}=\kappa_{x, 0} \gamma_{0}\left|B^{\delta}\right| \beta_{x, 1} \nabla \phi_{x}^{0}(\hat{y}) \cdot \nabla p_{x}(\hat{y})+\mathcal{O}\left(\delta^{2 N-1}\right)
$$

where

$$
\beta_{x, 1}:=\frac{\kappa_{x, 0}-\kappa_{x, 1}}{\kappa_{x, 1}+(N-1) \kappa_{x, 0}} .
$$


Similarly as in (106)-(108) we have

$$
\begin{aligned}
\int_{\Gamma_{\delta}} \kappa_{x, 0} \partial_{n} \xi_{0} p_{x} & =\gamma_{0} \kappa_{x, 0}(N-1)\left|B^{\delta}\right| \beta_{x, 1} \nabla \phi_{x}^{0}(\hat{y}) \cdot \nabla p_{x}(\hat{y}) \\
& +\gamma_{0}\left|B^{\delta}\right|\left(\mu_{x, 0}-\mu_{x, 1}\right) \phi_{x}^{0}(\hat{y}) p_{x}(\hat{y})+\mathcal{O}\left(\delta^{N+2}\right) .
\end{aligned}
$$

Gathering the previous results when $\gamma_{0} \neq 0$, we obtain

$$
\begin{aligned}
\mathcal{K}_{m, U}^{(0)} & =\mathcal{R} e\left(\int_{\Sigma} 2 \rho_{m} \psi_{m, U}^{(0)} \overline{\left(\rho \phi_{m}^{0}-\mathscr{M}\right)}\right)=\mathcal{R} e\left(\rho_{m} \int_{\Omega} \xi p_{m}\right) \\
& =\mathcal{R} e\left(\rho_{m} \kappa_{x, 0} N\left|B^{\delta}\right| \beta_{x, 1} \nabla \phi_{x}^{0}(\hat{y}) \cdot \nabla p_{x}(\hat{y})\right) \\
& +\mathcal{R} e\left(\rho_{m}\left|B^{\delta}\right|\left(\mu_{x, 0}-\mu_{x, 1}\right) \phi_{x}^{0}(\hat{y}) p_{x}(\hat{y})\right)+\mathcal{R}_{\mathcal{K}_{m, U}}
\end{aligned}
$$

where $\mathcal{R}_{\mathcal{K}_{m, U}}$ is obtained by collecting the remainders in (144), (145) and (146). We have $\mathcal{R}_{\mathcal{K}_{m, U}}:=\mathcal{O}\left(\delta^{N+2}\right)$. In the case $\gamma_{0}=0$ we note that all terms in (147) vanish except for the remainder.

Remark 1 Note that formula (147) is shorter as the general formula (110). The reason is that the term $f_{1}(\hat{y})-f_{0}(\hat{y})$ in formula $(110)$ is substituted by $q(\hat{y})-q(\hat{y})=0$ in (147) and thus vanishes.

\section{Topological derivative for FDOT}

Gathering the results of Section 4.3.1, and in particular using (129), (131) and (147) we obtain:

thm2 Theorem 2 We have the following expansion of the functional given by (124):

$$
\mathcal{J}\left(\Omega^{\delta}\right)=\mathcal{J}(\Omega)+\left|B^{\delta}\right| \mathcal{T}_{m}(\hat{y})+\mathcal{R}_{m}^{\delta}(\hat{y}) \text { as } \delta \searrow 0
$$

where $\mathcal{T}_{m}$ is the so-called topological derivative given by

$$
\begin{aligned}
\mathcal{T}_{m}(\hat{y})=\mathcal{R} e\left(\rho_{m}[\right. & N \kappa_{m, 0} \beta_{m, 1} \nabla \phi_{m}^{0}(\hat{y}) \cdot \nabla p_{m}(\hat{y}) \\
& +\left(\left(\mu_{m, 0}-\mu_{m, 1}\right) \phi_{m}^{0}(\hat{y})+\left(\gamma_{1}-\gamma_{0}\right) \phi_{x}^{0}(\hat{y})\right) p_{m}(\hat{y}) \\
+N & \kappa_{x, 0} \beta_{x, 1} \nabla \phi_{x}^{0}(\hat{y}) \cdot \nabla p_{x}(\hat{y}) \\
& \left.\left.+\left(\mu_{x, 0}-\mu_{x, 1}\right) \phi_{x}^{0}(\hat{y}) p_{x}(\hat{y})\right]\right)
\end{aligned}
$$

with $\beta_{j, 1}, j=m, x$, defined as

$$
\beta_{j, 1}:=\frac{\kappa_{j, 0}-\kappa_{j, 1}}{\kappa_{j, 1}+(N-1) \kappa_{j, 0}} .
$$

Under assumption (16)-(17), the remainder $\mathcal{R}_{m}^{\delta}(\hat{y})$ satisfies $\mathcal{R}_{m}^{\delta}(\hat{y})=\mathcal{O}\left(\delta^{N+2}\right)$. The states $\left(\phi_{m}^{0}, \phi_{x}^{0}\right)$ satisfy the forward system

$$
\begin{aligned}
-\operatorname{div}\left(\kappa_{x, 0} \nabla \phi_{x}^{0}\right)+\mu_{x, 0} \phi_{x}^{0}=q \quad \text { in } \Omega, & \text { (149) } \\
\kappa_{x, 0} \partial_{n} \phi_{x}^{0}+\rho_{x} \phi_{x}^{0}=0 & \text { on } \Sigma, \\
-\operatorname{div}\left(\kappa_{m, 0} \nabla \phi_{m}^{0}\right)+\mu_{m, 0} \phi_{m}^{0}=\gamma_{0} \phi_{x}^{0} \quad \text { in } \Omega, & \text { (150) } \\
\kappa_{m, 0} \partial_{n} \phi_{m}^{0}+\rho_{m} \phi_{m}^{0}=0 \quad \text { on } \Sigma, & \text { (151) }
\end{aligned}
$$

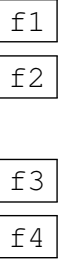


and the adjoints $p_{m}$ and $p_{x}$ solve

$$
\begin{aligned}
&-\operatorname{div}\left(\kappa_{m, 0} \nabla p_{m}\right)+\mu_{m, 0} p_{m}=0 \text { in } \Omega, \\
& \kappa_{m, 0} \partial_{n} p_{m}+\rho_{m} p_{m}=2 \overline{\left(\rho_{m} \phi_{m}^{0}-\mathscr{M}\right)} \text { on } \Sigma, \\
&-\operatorname{div}\left(\kappa_{x, 0} \nabla p_{x}\right)+\mu_{x, 0} p_{x}=\gamma_{0} p_{m} \quad \text { in } \Omega, \\
& \kappa_{x, 0} \partial_{n} p_{x}+\rho_{x} p_{x}=0 \quad \text { on } \Sigma .
\end{aligned}
$$

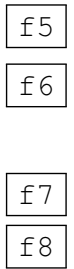

Remark 2 According to (129), (131), (132) and (147), the remainder $\mathcal{R}_{m}^{\delta}$ in (148) is related to the remainders in Section 4.3 .1 by

$$
\mathcal{R}_{m}^{\delta}=\mathcal{R}_{\mathcal{K}_{m, \phi}}^{\delta}+\mathcal{R}_{\mathcal{K}_{m, U}}^{\delta}+\mathcal{L}_{m}^{\delta}+\mathcal{K}_{m, U}^{(1)}
$$

For the numerical realization, according to Sections 4.2 and 4.3.1, we consider additional terms and we make use of the following expansion instead of (148)

$$
\mathcal{J}\left(\Omega^{\delta}\right)=\mathcal{J}(\Omega)+\left|B^{\delta}\right| \mathcal{T}(\hat{y})+\mathcal{L}_{0, m}^{\delta}(\hat{y})+\widetilde{\mathcal{R}}_{m}^{\delta}(\hat{y}) \quad \text { as } \delta \searrow 0
$$

where $\widetilde{\mathcal{R}}_{m}^{\delta}:=\mathcal{R}_{m}^{\delta}-\mathcal{L}_{0, m}^{\delta}(\hat{y})$. As discussed in the previous sections, the advantage of (157) compared to (148) is that it may be used in the entire domain $\Omega$ and corrects the boundary effect which was discussed in Section 3. Following (112), $\mathcal{L}_{0, m}^{\delta}$ in (157) is defined as

$$
\mathcal{L}_{0, m}^{\delta}:=\int_{\Sigma}\left|\rho^{m}\left(U_{m}+V_{m}^{(1)}\right)\right|^{2}
$$

where

$$
\begin{aligned}
& U_{m}=\frac{\delta^{N}}{|y-\hat{y}|^{N}} \beta_{m, 1} \nabla \phi_{m}^{0}(\hat{y}) \cdot(y-\hat{y}), \\
& V_{m}^{(1)}=\beta_{m, 2} \delta^{N} Q(|y-\hat{y}|) \\
& \quad \text { with } \beta_{m, 2}=\frac{1}{\kappa_{m, 0} N}\left[\left(\mu_{m, 0}-\mu_{m, 1}\right) \phi_{m}^{0}(\hat{y})+\left(\gamma_{1}-\gamma_{0}\right) \phi_{x}^{0}(\hat{y})\right]
\end{aligned}
$$

and for $y \neq \hat{y}$,

$$
Q(|y-\hat{y}|)= \begin{cases}-\ln (|y-\hat{y}|) & \text { for } N=2 \\ |y-\hat{y}|^{-1} & \text { for } N=3\end{cases}
$$

\section{Numerical results}

Numerically, the topological derivative may be realized in several different ways. We describe two options here. First, when the number of inclusions is unknown, then one may take a conservative approach by looking for one inclusion at a time. In this case, one computes the topological derivative and, according to the topological expansion, creates a small inclusion at a location where the topological derivative is most negative. This process is then iterated until the topological derivative is positive all over the domain $\Omega$. As an alternative to this approach, it has been observed that a more progressive step may be taken by inserting several inclusions or larger areas within a single iteration. This is achieved numerically by declaring the set of negative values of the topological derivative (or a subset thereof) an inclusion. In the case where several 
inclusions are introduced simultaneously, this procedure works well as long as the inclusions are of comparable size; otherwise small inclusions are typically "hidden" by large inclusions since the latter are usually detected first.

When considering topological and non-smooth modifications of a shape, then one faces a vast amount of possibilities, which may not be considered in a single paper. For instance, geometric modifications of different radii or shapes (including non-smooth shapes) for simultaneous inclusions, the case of several close or intersecting objects or inclusions very close to the boundary are all interesting cases which are not directly handled by our asymptotic expansions. Although asymptotic formulas may be theoretically obtained for each of these situations too, one has to consider their practical relevance from a numerical point of view. Indeed, more complex geometric changes may increase the computational burden considerably. Empirically it has turned out that it is usually beneficial to have a rough estimate of the topology, only, by using the topological derivative, and then to capture complex geometric features afterwards by applying shape sensitivity.

In order to keep the discussion of this paper focused, we concentrate on the case of a single inclusion and compare the result with the single step reconstruction approach of [16].

\subsection{Algorithm}

A common strategy when applying the topological derivative within a steepest descent framework is to create a small inclusion where the topological derivative is most negative. This is sometimes called the conservative approach with respect to the asymptotic expansion. Alternatively, a less conservative, but heuristic approach is to define the trial inclusion as a subset of the set of negative values of the topological derivative. This subset is determined by a user-specified threshold in $a \in(0,1)$.

As discussed in the previous sections, in the case of FDOT, instead of looking for the minimum of the topological derivative $\mathcal{T}(\hat{y})$ in $\Omega$ according to the expansion (148), we look for the minimum of the extended expansion $\left|B^{\delta}\right| \mathcal{T}(\hat{y})+\mathcal{L}_{0, m}^{\delta}(\hat{y})$ appearing in (157). However, this expression depends on $\delta$ which raises a number of questions concerning its appropriate numerical realization. At this point it appears that there are several possible approaches.

First method: We set

$$
\mathcal{T}_{m}^{\delta}(\hat{y}):=\left|B^{\delta}\right| \mathcal{T}(\hat{y})+\mathcal{L}_{0, m}^{\delta}(\hat{y})
$$

and fix an interval $I=[\underline{\delta}, \bar{\delta}]$ with user-specified constants $\underline{\delta}$ and $\bar{\delta}$. Then $I$ is divided into sub-intervals $\left[\delta_{i}, \delta_{i+1}\right], i=1, . ., n_{I-1}$, with $\delta_{1}=\underline{\delta}, \delta_{i}<\delta_{i+1}$ and $\delta_{n_{I}}=\bar{\delta}$. For $i$ increasing from 1 to $n_{I}$, we consider the trial inclusions

$$
\Omega_{1}^{(i)}:=\left\{x \in \Omega \mid \mathcal{T}_{m}^{\delta_{i}}(x)<a \min _{y \in \Omega} \mathcal{T}_{m}^{\delta_{i}}(y)\right\}
$$

unless $\Omega_{1}^{(i)}=\emptyset$, which happens when $\mathcal{T}_{m}^{\delta_{i}}(y) \geq 0$ for all $y \in \Omega$, or when $i=n_{I}$. We then choose $i$ for which $\mathcal{J}\left(\Omega \backslash \overline{\Omega_{1}^{(i)}}\right)$ is minimized. Obviously, the numerical cost of this operation depends on $n_{I}$.

Second method: We minimize first $\left|B^{\delta}\right| \mathcal{T}(\hat{y})+\mathcal{L}_{0, m}^{\delta}(\hat{y})$ with respect to $\delta$, within a given range $[\underline{\delta}, \bar{\delta}]$, and then with respect to the position $\hat{y}$. This is meaningful because 
the term $\min \left(\left|B^{\delta}\right| \mathcal{T}(\hat{y}), 0\right)$ is decreasing while $\mathcal{L}_{0, m}^{\delta}(\hat{y})$ is increasing with respect to $\delta$. Thus, we expect the existence of an optimal value $\delta_{o p t} \in[\underline{\delta}, \bar{\delta}]$. Consequently, we define the function

$$
\mathcal{T}_{m}(\hat{y}):=\min _{\delta \in[\underline{\delta}, \bar{\delta}]}\left(\left|B^{\delta}\right| \mathcal{T}(\hat{y})+\mathcal{L}_{0, m}^{\delta}(\hat{y})\right) .
$$

In numerical computation, $[\delta, \bar{\delta}]$ is divided into a user-defined number of sub-intervals. This discretization may be very fine and usually does not impose a heavy computational burden.

There are two approaches of how to use $\mathcal{T}_{m}(\hat{y}):(1)$ The first approach consists in finding the minimum of $\mathcal{T}_{m}(\cdot)$ and in obtaining a point $\bar{y}$. Then $\delta$ is given by the minimizing argument in (159). Hence, the domain is initialized by creating inclusions with centers $\bar{y}$ and respective radius $\delta$ (the shape of inclusions are balls, but "any" shape could be considered instead). (2) The second approach consists in choosing a threshold $a \in(0,1)$ and in initializing the domain $\Omega_{1}$ by

$$
\Omega_{1}:=\left\{x \in \Omega \mid \mathcal{T}_{m}(x)<a \min _{y \in \Omega} \mathcal{T}_{m}(y)\right\} .
$$

The first approach is completely automatized, but allows only to create inclusions of fixed shapes. The second approach, on the other hand, is more flexible in terms of the shape, and may therefore provide finer results. It, however, needs a user-defined parameter $a$ as an input quantity.

In this paper we use the first method which gave better results for the problem under investigation. The second method was also successfully employed for other inverse problems; see [25].

\subsection{Results}

In our tests, the domain $\Omega$ is a disk of center $\mathcal{O}$, the origin, and radius 15 . We use piecewise linear and globally continuous finite elements to solve the state equation (149)-(152) and the adjoint equation (153)-(156). The disk is discretized by an unstructured triangular mesh with 3,137 nodes and 6,016 elements. The values of the coefficients in (5)-(6) are chosen based on physical observations [1, 31, 32]. The values for the reduced scattering and absorption coefficients are $\mu_{s, x}^{\prime}=0.275 \mathrm{~mm}^{-1}$, $\mu_{a, x}=0.036 \mathrm{~mm}^{-1}, \mu_{s, m}^{\prime}=0.235 \mathrm{~mm}^{-1}$ and $\mu_{a, m}=0.029 \mathrm{~mm}^{-1}$ and the reflection coefficients $\rho_{x}=\rho_{m}=0.2$ are used. The flurophore's molar extinction coefficients are set to $\epsilon_{x}=8.35 \mathrm{~mm}^{-1} \mathrm{mM}^{-1}$ and $\epsilon_{m}=2.81 \mathrm{~mm}^{-1} \mathrm{mM}^{-1}$, its quantum efficiency is $\eta=0.016$ and its lifetime $\tau=1 \mathrm{~ns}$, which approximates indocyanine green. We take the frequency $\omega=0$. The true concentrations are $c_{0}=0 \mathrm{mM}$ and $c_{1}=10^{-2} \mathrm{mM}$. We use the first method described in the previous section. For the tests we take $\underline{\delta}=0.1$, $\bar{\delta}=2, a=0.1$ and $n_{I}=100$. The corresponding values of the parameters $\kappa_{j}, \mu_{j}, \gamma_{j}$, $j=x, m$ are given in Table 1 . We use 16 light sources (corresponding to $q$ in (1)) spaced equidistantly on the boundary.

First experiment: In Figure 1 we compare the reconstruction provided by our topological derivative based algorithm against a conventional reconstruction algorithm [16]. The latter is a single-step method which uses an adjoint technique to compute the sensitivity of the measurement data. A Tikhonov-type regularization term based on the $L^{2}$-norm of the fluorophore concentration is added to stabilize the inversion. For both algorithms we assume that the true values $\left(c_{0}, c_{1}\right)=\left(0,10^{-2}\right)$ are known. The left column in Figure 1 depicts the true inclusion. In the middle column we show the results 


\begin{tabular}{cccccc}
\hline & $\kappa_{j, 0}$ & $\kappa_{j, 1}$ & $\mu_{j, 0}$ & $\mu_{j, 1}$ & $\gamma_{0}$ \\
\hline$j=x$ & 1.6077 & 1.2674 & 0.036 & 0.1195 & 0 \\
\hline$j=m$ & 1.8939 & 1.7117 & 0.029 & 0.0571 & 0.0013 \\
\hline
\end{tabular}

Table 1: Numerical values of the coefficients appearing in (1)-(4) used in our experiments.

of the reconstruction using our topological derivative based concept. In the right column, the results using the single step reconstruction algorithm are shown. Due to the nature of our algorithm, we obtain a concentration with sharp interfaces. This is not the case with the single step reconstruction algorithm, as can be seen in Figure 1 where the concentration is a continuous function. The sharp interface can be obtained by thresholding the solution by means of a user-specified threshold-value. We observe that the reconstruction using the topological derivative is very precise and gives a more accurate position of the inclusion as the single step reconstruction, which typically exhibits a deviation towards the boundary. We also observe that the shape of the true inclusion does not affect the location of its reconstruction but, of course, the shape would still need to be reconstructed by subsequent shape optimization steps. This remark is valid for both algorithms. In our case, it would be possible to detect this shape using a shape sensitivity-based algorithm as in [24, 25] and the result in Figure 1 as initial guess.

Second experiment: In Figure 2 we test the robustness of the topological derivative based algorithm with respect to trial values of $\left(c_{0}, c_{1}\right)$. The left column in Figure 2 depicts the true inclusion. In the first row we show the results of the reconstruction when the exact value $c_{0}=0$ and various trial values of $c_{1}$ are used to compute the topological derivative, the exact value being $c_{1}=10^{-2}$. In the second row we take the exact value $c_{1}=10^{-2}$ and test different trial values of $c_{0}$. We observe that the topological derivative is quite stable with respect to the variation of the trial value $c_{1}$, and a little less stable with respect to $c_{0}$ (in the case $c_{0}=5.10^{-4}$, the reconstruction fails). Indeed the location of the inclusion is always well reconstructed, whereas the size of the reconstructed inclusion seems to depend on the trial values of $\left(c_{0}, c_{1}\right)$.

Third experiment: In Figures 3 and 4 we test the robustness of the topological derivative with respect to unknown perturbations of the parameters $\kappa_{x}, \mu_{x}, \kappa_{m}, \mu_{m}$. In this experiment the true coefficients $\tilde{\kappa}_{x}, \tilde{\mu}_{x}, \tilde{\kappa}_{m}, \tilde{\mu}_{m}$ used to compute the synthetic data are not constant in the background $\Omega_{0}$ and in the inclusion $\Omega_{1}$, as was the case in the previous examples. We deliberately compute the topological derivative with the erroneous functions $\kappa_{x}, \mu_{x}, \kappa_{m}, \mu_{m}$. The true inclusion, which we aim to reconstruct, is the concave object corresponding to the third line in Figure 1. In Figure 3, the coefficients $\kappa_{x}, \mu_{x}, \kappa_{m}, \mu_{m}$ are modified one at a time. For instance, in the left column the synthetic measurements are computed using $\tilde{\kappa}_{x}$, and the topological derivative using $\kappa_{x}$. In Figure 4 we use the coefficients $\tilde{\kappa}_{x}, \tilde{\mu}_{x}, \tilde{\kappa}_{m}, \tilde{\mu}_{m}$ simultaneously to produce the synthetic measurements. We observe that in all cases the location is precisely reconstructed, while the shape and size of the reconstruction may vary slightly.

Acknowledgements. The authors would like to thank the anonymous referees whose comments helped to improve the presentation of the paper. The authors acknowledge 

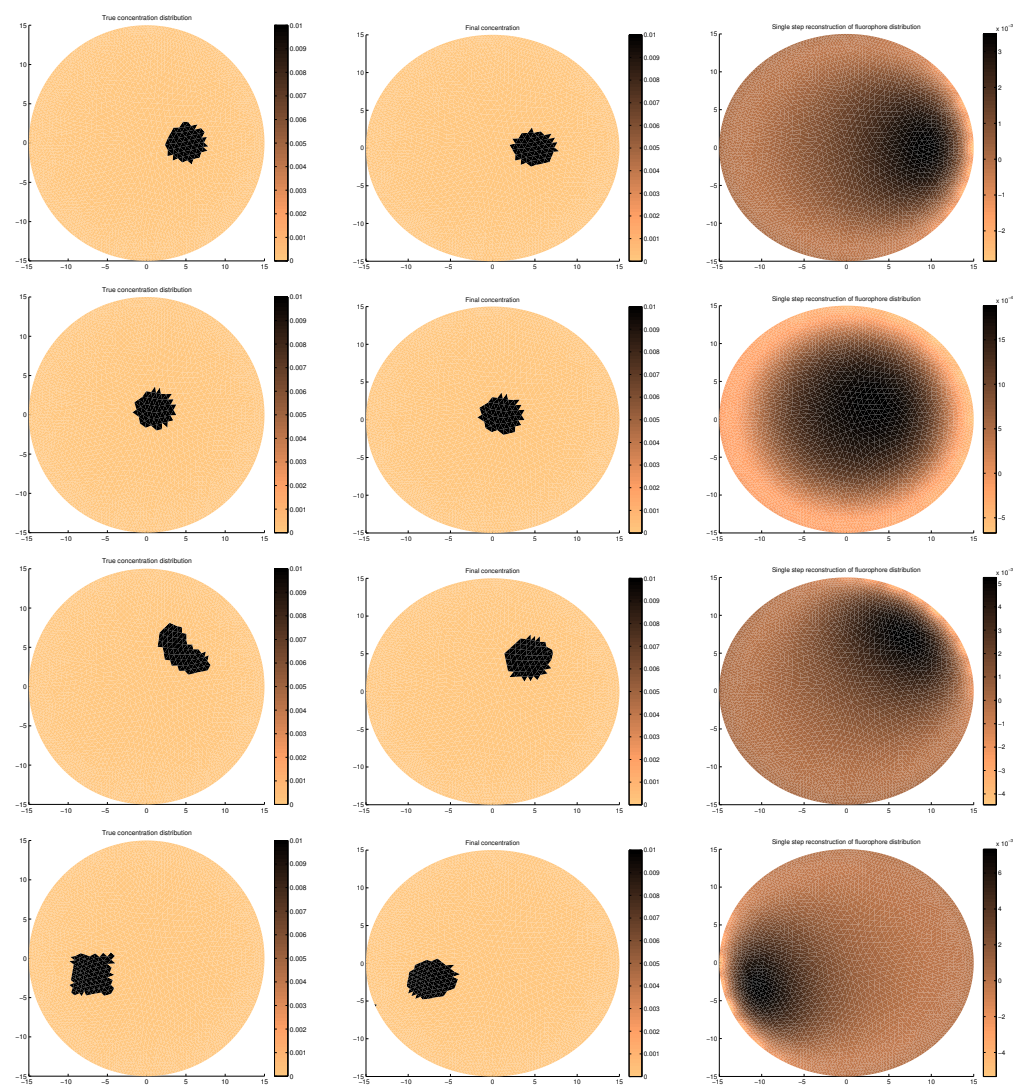

Figure 1: true inclusion (left column), reconstruction using the topological derivative (middle column), reconstruction using the single step algorithm (right column)

financial support by the Austrian Ministry of Science and Education and the Austrian Science Foundation FWF under START-grant Y305 "Interfaces and Free Boundaries" and the subproject "Freelevel" of the SFB F32 "Mathematical Optimization and Applications in Biomedical Sciences". They also acknowledge support from the DFG research center MATHEON and SPP1253 "Optimization with PDE's".

\section{References}

[1] G. Alexandrakis, F. R. Rannou, and A. F. Chatziioannou. Tomographic bioluminescence imaging by use of a combined optical-pet (opet) system: a computer simulation feasibility study. Physics in Medicine and Biology, 50:4225-4241, 2005.

Alvarez:09

[2] D. Álvarez, P. Medina, and M. Moscoso. Fluorescence lifetime imaging from timeresolved measurements using a shape-based approach. Opt. Express, 17(11):8843-8855, May 2009.

MR2888256

[3] H. Ammari, J. Garnier, V. Jugnon, and H. Kang. Stability and resolution analysis for a topological derivative based imaging functional. SIAM J. Control Optim., 50(1):48-76, 2012. 

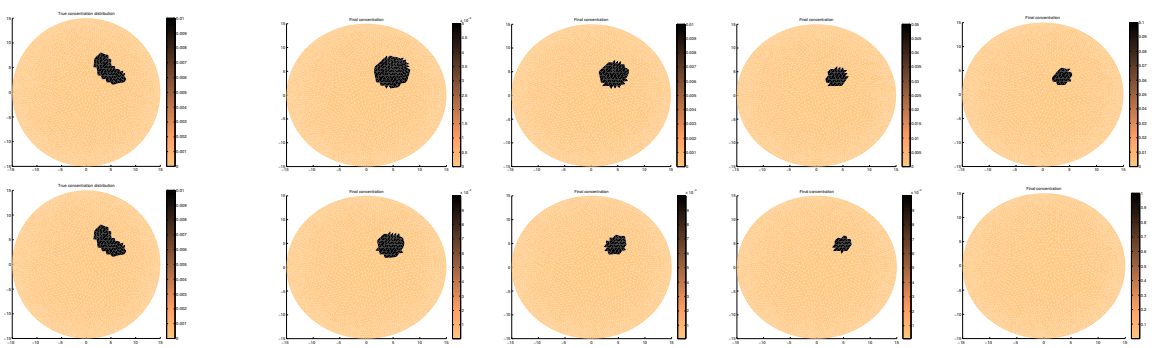

Figure 2: Second experiment. True inclusion (left column), reconstructed inclusion with trial values $c_{0}=0$ and $c_{1}=5.10^{-3}, 1.10^{-2}, 5.10^{-2}, 1.10^{-1}$ (first line from left to right), reconstructed inclusion with trial values $c_{0}=3.10^{-5}, 7.10^{-5}, 1.10^{-4}, 5.10^{-4}$ and $c_{1}=1.10^{-2}$ (second line from left to right).
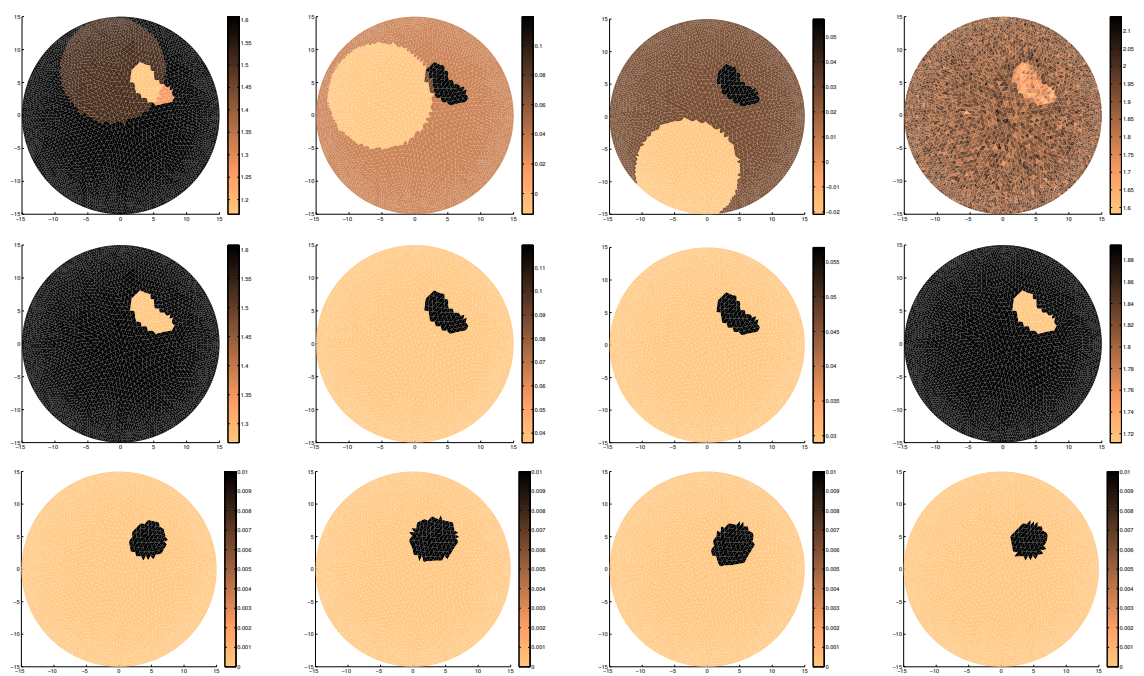

Figure 3: Third experiment. True coefficients $\tilde{\kappa}_{x}, \tilde{\mu}_{x}, \tilde{\kappa}_{m}, \tilde{\mu}_{m}$ (first line from left to right). Erroneous coefficients $\kappa_{x}, \mu_{x}, \kappa_{m}, \mu_{m}$ used deliberately to compute the topological derivative (second line from left to right). Corresponding reconstructions (third line).
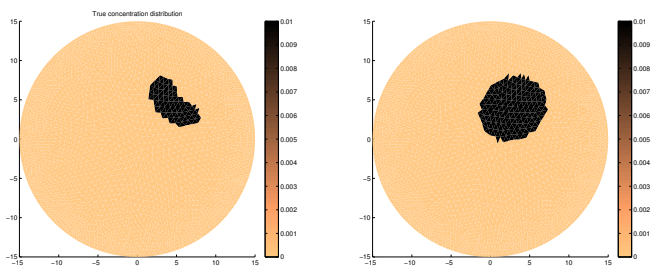

Figure 4: Third experiment. We use here simultaneously the coefficients $\tilde{\kappa}_{x}, \tilde{\mu}_{x}, \tilde{\kappa}_{m}, \tilde{\mu}_{m}$ appearing in Figure 3 to generate the synthetic data and the erroneous coefficients $\kappa_{x}, \mu_{x}, \kappa_{m}, \mu_{m}$ to compute the topological derivative. True inclusion (left), reconstruction (right). 
MR1969606

MR21 68949

MR2005296

MR2216306

MR2211064

arridge: 1999

MR2559100

MR2 421976

MR2517928

MR2425881

MR1 629995

MR2463668

efs:2010

Gannot 2004

MR1825864

MR2261265

hastings:mcmc_hm

MR2536481
[4] H. Ammari, E. Iakovleva, and S. Moskow. Recovery of small inhomogeneities from the scattering amplitude at a fixed frequency. SIAM J. Math. Anal., 34(4):882-900 (electronic), 2003.

[5] H. Ammari and H. Kang. Reconstruction of small inhomogeneities from boundary measurements, volume 1846 of Lecture Notes in Mathematics. Springer-Verlag, Berlin, 2004.

[6] H. Ammari and A. Khelifi. Electromagnetic scattering by small dielectric inhomogeneities. J. Math. Pures Appl. (9), 82(7):749-842, 2003.

[7] S. Amstutz. Topological sensitivity analysis for some nonlinear PDE system. J. Math. Pures Appl. (9), 85(4):540-557, 2006.

[8] S. Amstutz, I. Horchani, and M. Masmoudi. Crack detection by the topological gradient method. Control Cybernet., 34(1):81-101, 2005.

[9] S. R. Arridge. Optical tomography in medical imaging. Inverse Problems, 15:R41-R93, 1999.

[10] A. Ben Abda, M. Hassine, M. Jaoua, and M. Masmoudi. Topological sensitivity analysis for the location of small cavities in Stokes flow. SIAM J. Control Optim., 48(5):2871-2900, 2009/10.

[11] M. Bonnet. Inverse acoustic scattering by small-obstacle expansion of a misfit function. Inverse Problems, 24(3):035022, 27, 2008.

[12] M. Bonnet. Higher-order topological sensitivity for 2-D potential problems. Application to fast identification of inclusions. Internat. J. Solids Structures, 46(11-12):2275-2292, 2009.

[13] A. Carpio and M.-L. Rapún. Solving inhomogeneous inverse problems by topological derivative methods. Inverse Problems, 24(4):045014, 32, 2008.

[14] D. J. Cedio-Fengya, S. Moskow, and M. S. Vogelius. Identification of conductivity imperfections of small diameter by boundary measurements. Continuous dependence and computational reconstruction. Inverse Problems, 14(3):553-595, 1998.

[15] I. Chikichev and B. B. Guzina. Generalized topological derivative for the Navier equation and inverse scattering in the time domain. Comput. Methods Appl. Mech. Engrg., 197(4950):4467-4484, 2008.

[16] H. Egger, M. Freiberger, and M. Schlottbom. On forward and inverse models in fluorescence diffuse optical tomography. Inverse Problems and Imaging, 4(3):411-427, 2010.

[17] H. Eschenauer, V. Kobelev, and A. Schumacher. Bubble method for topology and shape optimization of structures. 8:42-51, 1994.

[18] G. R. Feijoo. A new method in inverse scattering based on the topological derivative. Inverse Problems, 20(6):1819-1840, 2004.

[19] M. Freiberger, H. Egger, and H. Scharfetter. Nonlinear inversion schemes for fluorescence optical tomography. IEEE Transactions on Biomedical Engineering, 57(11):2723-2729, 2010 .

[20] I. Gannot, I. Ron, F. Hekmat, V. Chernomordik, and A. Gandjbakhche. Functional optical detection based on ph dependent fluorescence lifetime. Lasers Surg Med, 35(5):342-348, 2004.

[21] S. Garreau, P. Guillaume, and M. Masmoudi. The topological asymptotic for PDE systems: the elasticity case. SIAM J. Control Optim., 39(6):1756-1778 (electronic), 2001

[22] B. B. Guzina and M. Bonnet. Small-inclusion asymptotic of misfit functionals for inverse problems in acoustics. Inverse Problems, 22(5):1761-1785, 2006.

[23] W. K. Hastings. Monte carlo sampling methods using markov chains and their applications. Biometrika, 57:97-109, 1970.

[24] M. Hintermüller and A. Laurain. Electrical impedance tomography: from topology to shape. Control Cybernet., 37(4):913-933, 2008. 
HiLaNo [25] M. Hintermüller, A. Laurain, and A. A. Novotny. Second-order topological expansion for electrical impedance tomography. Adv. Comput. Math., 2010.

1073.68095 [26] M. Hintermüller and W. Ring. A second order shape optimization approach for image segmentation. SIAM J. Appl. Math., 64(2):442-467, 2003.

Ilin [27] A. M. Il'in. Matching of asymptotic expansions of solutions of boundary value problems, volume 102 of Transl. Math. Monog. American Mathematical Society, Providence, RI, 1992.

jiang:1998

[28] H. Jiang. Frequency-domain fluorescent diffusion tomography: A finite-element-based algorithm and simulations. Appl. Opt., 37:5337-5343, 1998.

joshi:2004

[29] A. Joshi, W. Bangerth, and W. M. Sevick-Muraca. Adaptive finite element based tomography for fluorescence optical imaging in tissue. Opt. Express, 12:5402-5417, 2004.

Joshi2004

keijzer: 1988

[30] A. Joshi, W. Bangerth, and W. M. Sevick-Muraca. Adaptive finite element based tomography for fluorescence optical imaging in tissue. Opt. Express, 12:5402-5417, 2004.

[31] M. Keijzer, W. M. Star, and P. R. M. Storchi. Optical diffusion in layered media. Applied Optics, 27:1820-1824, 1988.

landsman: 1976

[32] M. L. Landsman, G. Kwant, G. A. Mook, and W. G. Zijlstra. Light-absorbing properties, stability, and spectral stabilization of indocyanine green. J Appl Physiol, 40(4):575-583, 1976.

Longmuir1976 [33] I. S. Longmuir and J. A. Knopp. Measurement of tissue oxygen with a fluorescent probe. J Appl Physiol, 41(4):598-602, 1976.

MaNaPl [34] V. G. Maz'ya, S. A. Nazarov, and B. A. Plamenevski1̌. Asimptotika reshenii ellipticheskikh kraevykh zadach pri singulyarnykh vozmushcheniyakh oblasti(Russian) [Asymptotic behavior of solutions of elliptic boundary value problems under singular perturbations of the domain]. Tbilis. Gos. Univ. Inst. Prikl. Mat., Tbilisi, 1981. With Georgian and English summaries.(German translation: Asymptotische Theorie elliptischer Randwertaufgaben in singulär gestörten Gebieten. 1. Berlin: Akademie-Verlag. 1991. 432 S.) (English translation: Asymptotic theory of elliptic boundary value problems in singularly perturbed domains. Vol. 1. Basel: Birkhäuser Verlag, 2000. 435 p.).

Mordon1992 [35] S. Mordon, V. Maunoury, J. M. Devoisselle, Y. Abbas, and D. Coustaud. Characterization of tumorous and normal tissue using a ph-sensitive fluorescence indicator (5,6carboxyfluorescein) in vivo. J Photochem Photobiol B, 13(3-4):307-314, May 1992.

reynolds:1997 [36] J. S. Reynolds, T. L. Troy, and E. M. Sevick-Muraca. Multipixel techniques for frequencydomain photon migration imaging. Biotechnol. Prog., 13:669-690, 1997.

roy: 1999

[37] R. Roy and E. M. Sevick-Muraca. Truncated Newton's optimization scheme for absorption and fluorescence optical tomography: Part I theory and formulation. Optics Express, 4:353-371, 1999.

schweiger:2006

[38] M. Schweiger, S. R. Arridge, O. Dorn, Z. Zacharopoulos, and V. Kolehmainen. Reconstructing absorption and diffusion shape profiles in optical tomography by a level set method. Optics Letters, 31:471-473, 2006.

schweiger:2005

Shives2002

[39] M. Schweiger, S. R. Arridge, and I. Nissilä. Gauss-Newton method of image reconstruction in diffuse optical tomography. Phys. Med. Biol., 50:2365 - 2386, 2005.

[40] E. Shives, Y. Xu, and H. Jiang. Fluorescence lifetime tomography of turbid media based on an oxygen-sensitive dye. Opt. Express, 10:1557-1562, 2002.

MR1691940 [41] J. Sokołowski and A. Żochowski. On the topological derivative in shape optimization. SIAM J. Control Optim., 37(4):1251-1272 (electronic), 1999.

MR1215733 [42] J. Sokołowski and J.-P. Zolésio. Introduction to shape optimization, volume 16 of Springer Series in Computational Mathematics. Springer-Verlag, Berlin, 1992. Shape sensitivity analysis.

MR0010746 [43] G. N. Watson. A Treatise on the Theory of Bessel Functions. Cambridge University Press, Cambridge, England, 1944. 


\section{Appendix}

\section{Computation of $\phi_{1}^{(k)}$ and $\widetilde{\phi}_{1}$}

In order to compute $\phi_{1}^{(k)}, k=0,1,2$, from (39) in a compact way, we look for the solution of the generic problem

$$
\begin{array}{rlll}
-a \Delta \Xi+b \Xi & =\alpha & \text { in } B^{\delta}, & \text { (160) } 12.1 \\
\Xi=\beta & \text { on } \Gamma^{\delta}, & \text { (161) } & 12.2
\end{array}
$$

where $a, b$ are positive constants and $\alpha, \beta$ denote functions. The Laplacian is given by

$$
\begin{aligned}
& \Delta=\partial_{r}^{2}+r^{-1} \partial_{r}+r^{-2} \partial_{\theta}^{2}, \\
& \Delta=\partial_{r}^{2}+2 r^{-1} \partial_{r}+r^{-2}(\sin \varphi)^{-2} \partial_{\theta}^{2}+r^{-2}(\tan \varphi)^{-1} \partial_{\varphi}+r^{-2} \partial_{\varphi}^{2},
\end{aligned}
$$

in polar $(r, \theta)(N=2)$ and spherical $(r, \theta, \varphi)(N=3)$ coordinates, respectively, with $r=|x|$.

Sinusoidal right-hand side: In two dimensions we may assume that $\alpha$ and $\beta$ are sinusoidal functions $\sin k \theta, \cos k \theta, k \in \mathbb{N}$. Therefore we may separate the variables and we consider functions of the type

$$
\alpha(r, \theta)=s_{\alpha}(r) t(\theta), \quad \beta(r, \theta)=s_{\beta}(r) t(\theta) \quad \Xi(r, \theta)=s(r) t(\theta) .
$$

Without loss of generality, we may assume that $t(\theta)=\sin (k \theta)$ or $\cos (k \theta)$ for some given $k \in \mathbb{N}$. We then have $t^{\prime \prime}(\theta)=-k^{2} t(\theta)$ In this case, (160)-(161) becomes

$$
\begin{aligned}
-a\left(r^{2} \partial_{r}^{2}+r \partial_{r}\right) \Xi+\left(r^{2} b+a k^{2}\right) \Xi & =\alpha r^{2} \quad \text { for }(r, \theta) \in(0, \delta) \times[0,2 \pi], \\
\partial_{r} \Xi(0, \theta)=0, \quad \Xi(\delta, \theta) & =\beta(\delta, \theta),
\end{aligned}
$$

or, upon separating the variables,

$$
\begin{aligned}
-a r^{2} s^{\prime \prime}(r)-a r s^{\prime}(r)+\left(r^{2} b+a k^{2}\right) s(r) & =s_{\alpha}(r) r^{2} \quad \text { for } r \in(0, \delta), \\
s^{\prime}(0) & =0, \quad s(\delta)=s_{\beta}(\delta) .
\end{aligned}
$$

The solutions to the homogeneous problem associated to (164)-(165) are given by

$$
s(r):=c_{1} J_{k}(\lambda r)+c_{2} Y_{k}(\lambda r), \quad \text { for } N=2,
$$

where $J_{k}$ and $Y_{k}$ are Bessel functions of the first and second kind [43], respectively, and $\lambda=(-b / a)^{1 / 2}$. We actually have $c_{2}=0$ due to $Y_{k}$ being singular at $r=0$. Therefore, we obtain a solution of the form

$$
\Xi(r, \theta)=c_{1} s_{k}(\lambda r) t(\theta)+s_{p}(r) t(\theta),
$$

where $s_{k}=J_{k}$ and $s_{p}$ is a particular solution. One checks that $s_{k}^{\prime}(0)=0$ for all $k \in \mathbb{N}$. Using the boundary condition (165) we get

$$
c_{1}=\left[s_{\beta}(\delta)-s_{p}(\delta)\right] s_{k}(\lambda \delta)^{-1} .
$$

In three dimensions we seek a solution similar to (167) but using spherical coordinates and functions

$$
s(r):=c_{1} j_{k}(\lambda r)+c_{2} y_{k}(\lambda r),
$$


where $j_{k}$ and $y_{k}$ are spherical Bessel functions [43]. As in two dimensions we have $c_{2}=0$. In order to keep the paper self-contained we display here the Bessel functions involved in (166) and (169).

$$
J_{k}(r)=\sum_{l=0}^{\infty} \frac{(-1)^{l}}{l !(l+k) !}\left(\frac{r}{2}\right)^{2 l+k}, \quad j_{k}(r)=\sqrt{\pi /(2 r)} J_{n+1 / 2}(r) .
$$

Computation of $\phi_{1}^{(0)}$ : Refering back to (27),(29) we specify the values of $a, b, \alpha, \beta$ and $k$ in (160)-(161) as $\Xi=\phi_{1}^{(0)}, a=\kappa_{1}, b=\mu_{1}, \alpha=f_{1}(\hat{y}), \beta=\phi^{0}(\hat{y})$ and $k=0$. Since $\alpha$ and $\beta$ are constants we obtain (50). In three dimensions, it is easy to check that $\phi_{1}^{(0)}$ is also expressed by (50). Thus, owing to the expansions

$$
\begin{array}{ll}
s_{0}(y)=J_{0}(y)=1-\frac{y^{2}}{4}+\mathcal{O}\left(y^{4}\right), & \text { for } N=2, \\
s_{0}(y)=j_{0}(y)=1-\frac{y^{2}}{6}+\mathcal{O}\left(y^{4}\right), & \text { for } N=3,
\end{array}
$$

as $y \rightarrow 0$ and using $\partial_{n}=-\partial_{r}$ on $\Gamma^{\delta}$ and for $N=2,3$, the normal derivative of $\phi_{1}^{(0)}$ is given by (53).

Computation of $\phi_{1}^{(1)}$ : In the same way, taking $\Xi=\phi_{1}^{(1)}, \alpha=\nabla f_{1}(\hat{y}) \cdot(y-\hat{y})$, $\beta=\nabla \phi^{0}(\hat{y}) \cdot(y-\hat{y}), a=\kappa_{1}, b=\mu_{1}$ and $k=1$, we obtain (51) where we use $y-\hat{y}=-\delta n$ on $\Gamma^{\delta}$. In dimension $N=3$, we check that $\phi_{1}^{(1)}$ is also given by (51). Since

$$
\begin{array}{ll}
s_{1}(y)=J_{1}(y)=-J_{0}^{\prime}(y)=\frac{y}{2}+\mathcal{O}\left(y^{3}\right) & \text { for } N=2, \\
s_{1}(y)=j_{1}(y)=-j_{0}^{\prime}(y)=\frac{y}{3}+\mathcal{O}\left(y^{3}\right) & \text { for } N=3,
\end{array}
$$

as $y \rightarrow 0$, respectively. For $N=2,3$, we have

$$
\partial_{n} \phi_{1}^{(1)}(\delta)=-c_{1}^{(1)} \cdot n \lambda s_{1}^{\prime}(\lambda \delta)+\frac{\nabla f_{1}(\hat{y}) \cdot n}{\mu_{1}} .
$$

Using the previous asymptotic expansions we get as $\delta \rightarrow 0$ :

$$
\begin{aligned}
\partial_{n} \phi_{1}^{(1)}(\delta) & =\nabla \phi^{0}(\hat{y}) \cdot n-\frac{\nabla f_{1}(\hat{y}) \cdot n}{\mu_{1}}+\frac{\nabla f_{1}(\hat{y}) \cdot n}{\mu_{1}}+\mathcal{O}\left(\delta^{2}\right) \\
& =\nabla \phi^{0}(\hat{y}) \cdot n+\mathcal{O}\left(\delta^{2}\right) .
\end{aligned}
$$

Computation of $\phi_{1}^{(2)}$ : In the case of $\phi_{1}^{(2)}$, the solution of the homogeneous problem associated with (164)-(165) is similar to the previous cases, but the particular solution is obtained in a different way. Indeed, using (166), we choose

$$
\alpha=\frac{1}{2} D^{2} f_{1}(\hat{y}) \cdot(y-\hat{y})^{2}, \quad \beta=\frac{1}{2} D^{2} \phi(\hat{y}) \cdot(y-\hat{y})^{2} .
$$

When $\alpha$ is harmonic, as for $\phi_{1}^{(0)}$ and $\phi_{1}^{(1)}$, the particular solution may be deduced straightforwardly. In the case of $\phi_{1}^{(2)}$, we have $\Delta \alpha=\Delta f_{1}(\hat{y}) \neq 0$, in general. In this case the particular solution is given by

$$
\frac{1}{2 \mu_{1}} D^{2} f_{1}(\hat{y}) \cdot(y-\hat{y})^{2}+\frac{\kappa_{1}}{\left(\mu_{1}\right)^{2}} \Delta f_{1}(\hat{y}) .
$$


For obtaining the solution of the homogeneous problem in dimension $N=2$, we decompose $\alpha$ and $\beta$ as

$$
\begin{aligned}
& \alpha=\frac{1}{2}\left[\frac{\Delta f_{1}(\hat{y})}{2}+\frac{\partial_{1}^{2} f_{1}(\hat{y})-\partial_{2}^{2} f_{1}(\hat{y})}{2} \cos (2 \theta)+\partial_{12}^{2} f_{1}(\hat{y}) \sin (2 \theta)\right] r^{2}, \\
& \beta=\frac{1}{2}\left[\frac{\Delta \phi(\hat{y})}{2}+\frac{\partial_{1}^{2} \phi(\hat{y})-\partial_{2}^{2} \phi(\hat{y})}{2} \cos (2 \theta)+\partial_{12}^{2} \phi(\hat{y}) \sin (2 \theta)\right] r^{2} .
\end{aligned}
$$

Following the general form (167) and using the particular solution (171), the solution is then given by

$$
\begin{aligned}
\phi_{1}^{(2)} & =c_{1}^{(2)} s_{0}(\lambda r)+c_{1}^{(2 c)} s_{4}(\lambda r) \cos (2 \theta)+c_{1}^{(2 s)} s_{4}(\lambda r) \sin (2 \theta) \\
& +\frac{1}{2 \mu_{1}} D^{2} f_{1}(\hat{y}) \cdot(y-\hat{y})^{2}+\frac{\kappa_{1}}{\left(\mu_{1}\right)^{2}} \Delta f_{1}(\hat{y})
\end{aligned}
$$

with

$$
\begin{aligned}
c_{1}^{(2)} & =\left[\frac{\delta^{2}}{4} \Delta \phi^{0}(\hat{y})-\frac{\delta^{2}}{4 \mu_{1}} \Delta f_{1}(\hat{y})-\frac{\kappa_{1}}{\left(\mu_{1}\right)^{2}} \Delta f_{1}(\hat{y})\right] s_{0}(\lambda \delta)^{-1}, \\
c_{1}^{(2 c)} & =\left[\frac{\delta^{2}}{4}\left(\partial_{1}^{2}-\partial_{2}^{2}\right)\left(\phi^{0}-\frac{f_{1}}{\mu_{1}}\right)(\hat{y})\right] s_{4}(\lambda \delta)^{-1}, \\
c_{1}^{(2 s)} & =\left[\frac{\delta^{2}}{2} \partial_{12}^{2}\left(\phi^{0}-\frac{f_{1}}{\mu_{1}}\right)(\hat{y})\right] s_{4}(\lambda \delta)^{-1},
\end{aligned}
$$

where we also use (168). For a given function $\Xi$, we have the relation

$$
\left[\cos (2 \theta)\left(\partial_{1}^{2}-\partial_{2}^{2}\right)+2 \sin (2 \theta) \partial_{12}^{2}\right](\Xi)(\hat{y})=-\frac{\Delta \Xi(\hat{y})}{N}+D^{2} \Xi(\hat{y}) \cdot(n)^{2} .
$$

Therefore, $\phi_{1}^{(2)}$ may be rewritten in the general form (52). One checks that (52) is also a solution for $N=3$. The normal derivative on $\Gamma^{\delta}$ is given by

$$
\begin{aligned}
\partial_{n} \phi_{1}^{(2)} & (\delta)=-c_{1}^{(2)} \lambda s_{0}^{\prime}(\lambda \delta)-\frac{\delta}{\mu_{1}} D^{2} f_{1}(\hat{y}) \cdot(n)^{2} \\
& -\frac{\delta^{2}}{4} \lambda s_{4}^{\prime}(\lambda \delta) s_{4}(\lambda \delta)^{-1}\left[-\frac{1}{N} \Delta\left(\phi^{0}-\frac{f_{1}}{\mu_{1}}\right)(\hat{y})+D^{2}\left(\phi^{0}-\frac{f_{1}}{\mu_{1}}\right)(\hat{y}) \cdot(n)^{2}\right] .
\end{aligned}
$$

The Bessel functions $J_{4}$ and $j_{4}$ admit the following expansions:

$$
\begin{aligned}
& s_{4}(y)=J_{4}(y)=\frac{1}{\Gamma(5)}\left(\frac{y}{2}\right)^{4}+\mathcal{O}\left(y^{6}\right) \quad \text { for } N=2, \\
& s_{4}(y)=j_{4}(y)=\frac{\sqrt{\pi}}{2 \Gamma(5+1 / 2)}\left(\frac{y}{2}\right)^{4}+\mathcal{O}\left(y^{6}\right) \quad \text { for } N=3,
\end{aligned}
$$

as $y \rightarrow 0$, respectively, where $\Gamma$ denotes the Gamma function. Finally, for the normal derivative we obtain (55) as $\delta \rightarrow 0$.

Computation of $\widetilde{\phi}_{1}$ : Using $f_{1}$ and $\phi_{0} \in \mathcal{C}^{4}(\Omega)$ we have the Taylor expansions at $\hat{y} \in \Omega$ :

$$
\begin{gathered}
\widetilde{\phi}^{0}(y)=\frac{1}{3 !} D^{3} \phi^{0}(\hat{y}) \cdot(y-\hat{y})^{3}+\frac{1}{4 !} D^{4} \phi^{0}\left(\hat{y}_{\phi}\right) \cdot(y-\hat{y})^{4}, \\
\widetilde{f}_{1}(y)=\frac{1}{3 !} D^{3} f_{1}(\hat{y}) \cdot(y-\hat{y})^{3}+\frac{1}{4 !} D^{4} \phi^{0}\left(\hat{y}_{f}\right) \cdot(y-\hat{y})^{4},
\end{gathered}
$$


where $\hat{y}_{f}, \hat{y}_{\phi} \in B(\hat{y}, y)$. To prove the estimate for $\widetilde{\phi}_{1}$ we decompose it as $\widetilde{\phi}_{1}=$ $\phi_{1}^{(3)}+\phi_{1}^{(4)}$ where $\phi_{1}^{(3)}$ is defined as the solution of (40)-(41) with the right-hand sides

$$
\alpha=\frac{1}{3 !} D^{3} f_{1}(\hat{y}) \cdot(y-\hat{y})^{3}, \quad \beta=\frac{1}{3 !} D^{3} \phi(\hat{y}) \cdot(y-\hat{y})^{3},
$$

and with the obvious definition of $\phi_{1}^{(4)}$. In the case of $\phi_{1}^{(3)}$, the solution of the homogeneous problem associated with (164)-(165) is similar to the previous cases, but the particular solution is

$$
\frac{\alpha}{\mu_{1}}+\frac{\kappa_{1}}{\left(\mu_{1}\right)^{2}} \Delta \alpha .
$$

To solve the solution of the homogeneous problem in dimension $N=2$, we use

$$
\begin{aligned}
& \alpha=\frac{r^{3}}{3 !}\left[A_{c}^{f} \cos \theta+A_{s}^{f} \sin \theta+A_{3 c}^{f} \cos (3 \theta)+A_{3 s}^{f} \sin (3 \theta)\right], \\
& \beta=\frac{r^{3}}{3 !}\left[A_{c}^{\phi} \cos \theta+A_{s}^{\phi} \sin \theta+A_{3 c}^{\phi} \cos (3 \theta)+A_{3 s}^{\phi} \sin (3 \theta)\right],
\end{aligned}
$$

with

$$
\begin{aligned}
A_{c}^{f} & =-\frac{3}{4} \partial_{1} \Delta f_{1}(\hat{y}), & A_{s}^{f} & =\frac{3}{4} \partial_{2} \Delta f_{1}(\hat{y}), \\
A_{3 c}^{f} & =\frac{1}{4} \partial_{1}^{3} f_{1}-\frac{3}{4} \partial_{122}^{3} f_{1}, & A_{3 s}^{f} & =-\frac{1}{4} \partial_{2}^{3} f_{1}+\frac{3}{4} \partial_{112}^{3} f_{1},
\end{aligned}
$$

and the same definition for $A_{c}^{\phi}, A_{s}^{\phi}, A_{3 c}^{\phi}, A_{3 s}^{\phi}$ with $\phi$ instead of $f_{1}$. Following the general form (167) and using the particular solution (173), the solution is

$$
\begin{aligned}
\phi_{1}^{(3)}= & c_{1}^{(c)} s_{1}(\lambda r) \cos \theta+c_{1}^{(s)} s_{1}(\lambda r) \sin \theta \\
& +c_{1}^{(3 c)} s_{9}(\lambda r) \cos (3 \theta)+c_{1}^{(3 s)} s_{9}(\lambda r) \sin (3 \theta)+\frac{\alpha}{\mu_{1}}+\frac{\kappa_{1}}{\left(\mu_{1}\right)^{2}} \Delta \alpha
\end{aligned}
$$

with

$$
\begin{aligned}
c_{1}^{(c)} & =\left[\frac{\delta^{3}}{3 !}\left(A_{c}^{\phi}-\frac{A_{c}^{f}}{\mu_{1}}\right)+\delta \frac{4 \kappa_{1}}{3\left(\mu_{1}\right)^{2}} A_{c}^{f}\right] s_{1}(\lambda \delta)^{-1}, \\
c_{1}^{(s)} & =\left[\frac{\delta^{3}}{3 !}\left(A_{s}^{\phi}-\frac{A_{s}^{f}}{\mu_{1}}\right)-\delta \frac{4 \kappa_{1}}{3\left(\mu_{1}\right)^{2}} A_{s}^{f}\right] s_{1}(\lambda \delta)^{-1}, \\
c_{1}^{(3 c)} & =\left[\frac{\delta^{3}}{3 !}\left(A_{3 c}^{\phi}-\frac{A_{3 c}^{f}}{\mu_{1}}\right)\right] s_{9}(\lambda \delta)^{-1}, \quad c_{1}^{(3 s)}=\left[\frac{\delta^{3}}{3 !}\left(A_{3 s}^{\phi}-\frac{A_{3 s}^{f}}{\mu_{1}}\right)\right] s_{9}(\lambda \delta)^{-1} .
\end{aligned}
$$

The computation of the derivative gives

$$
\begin{aligned}
\partial_{n} \phi_{1}^{(3)}(\delta)= & -c_{1}^{(c)} \lambda s_{1}^{\prime}(\lambda \delta) \cos \theta-c_{1}^{(s)} \lambda s_{1}^{\prime}(\lambda \delta) \sin \theta \\
& -c_{1}^{(3 c)} \lambda s_{9}^{\prime}(\lambda \delta) \cos (3 \theta)-c_{1}^{(3 s)} \lambda s_{9}^{\prime}(\lambda \delta) \sin (3 \theta) \\
& -\frac{\delta^{2}}{2 \mu_{1}}\left[A_{c}^{f} \cos \theta+A_{s}^{f} \sin \theta+A_{3 c}^{f} \cos (3 \theta)+A_{3 s}^{f} \sin (3 \theta)\right] \\
& +\frac{4 \kappa_{1}}{3\left(\mu_{1}\right)^{2}}\left[A_{c}^{f} \cos \theta-A_{s}^{f} \sin \theta\right] .
\end{aligned}
$$


Using (170) and writing asymptotic expansions as $\delta \rightarrow 0$ we get $\partial_{n} \phi_{1}^{(3)}(\delta)=\mathcal{O}\left(\delta^{2}\right)$. We also get this estimate in dimension $N=3$.

Estimate for $\phi_{1}^{(4)}$ : Let $\alpha=(4 !)^{-1} D^{4} f_{1}\left(y_{f}\right) \cdot(y-\hat{y})^{4}$ and $\beta=(4 !)^{-1} D^{4} \phi^{0}\left(y_{\phi}\right)$. $(y-\hat{y})^{4}$. According to standard elliptic regularity results, we have

$$
\left\|\phi_{1}^{(4)}\right\|_{H^{3}\left(B_{\delta}\right)} \leq c\left(\|\alpha\|_{H^{1}\left(B_{\delta}\right)}+\|\beta\|_{H^{2+1 / 2}\left(\Gamma_{\delta}\right)}\right) .
$$

For the first norm on the right-hand side, using the boundedness of $f_{1}$ and its derivative on $B^{\delta}$ we have

$$
\|\alpha\|_{H^{1}\left(B_{\delta}\right)}^{2}=\int_{B^{\delta}}\left[\sum_{|\gamma|=1} D^{\gamma} \frac{1}{4 !} D^{4} f_{1}\left(y_{f}\right) \cdot(y-\hat{y})^{4}\right]^{2} \leq c \int_{B^{\delta}} r^{6} \leq c \delta^{6+N} .
$$

For the norm of $\beta$ we have

$$
\begin{aligned}
\|\beta\|_{H^{2+1 / 2}\left(\Gamma_{\delta}\right)}^{2} & \leq c\|\beta\|_{H^{3}\left(B_{\delta}\right)}^{2} \leq c\left\|\sum_{|\gamma|=3} D^{\gamma} \beta\right\|_{L^{2}\left(B_{\delta}\right)}^{2} \\
& \leq \int_{B^{\delta}}\left[\sum_{|\gamma|=3} D^{\gamma} \frac{1}{4 !} D^{4} \phi^{0}\left(y_{\phi}\right) \cdot(y-\hat{y})^{4}\right]^{2} \leq c \delta^{2+N} .
\end{aligned}
$$

Thus we get

$$
\|\beta\|_{H^{2+1 / 2}\left(\Gamma_{\delta}\right)} \leq c \delta^{1+N / 2} \text { and }\left\|\phi_{1}^{(4)}\right\|_{H^{3}\left(B_{\delta}\right)} \leq c \delta^{3+N / 2}+c \delta^{1+N / 2} .
$$

For the gradient this implies $\left\|\nabla \phi_{1}^{(4)}\right\|_{H^{2}\left(B_{\delta}\right)} \leq c \delta^{3+N / 2}+c \delta^{1+N / 2}$. According to standard Sobolev imbeddings, $H^{2}\left(B_{\delta}\right)$ is imbedded in $L^{\infty}\left(B_{\delta}\right)$ for $N=2$ and $N=3$, yielding

$$
\left\|\nabla \phi_{1}^{(4)}\right\|_{L^{\infty}\left(B_{\delta}\right)} \leq c \delta^{1+N / 2} \Longrightarrow\left|\partial_{n} \phi_{1}^{(4)}(\delta)\right| \leq c \delta^{1+N / 2} .
$$

Therefore, with $\widetilde{\phi}_{1}=\phi_{1}^{(3)}+\phi_{1}^{(4)}$ we get the estimate (56). 